\title{
Logarithmic Schrödinger equation with quadratic potential
}

\author{
Rémi Carles and Guillaume Ferriere
}

\begin{abstract}
We analyze dynamical properties of the logarithmic Schrödinger equation under a quadratic potential. The sign of the nonlinearity is such that it is known that in the absence of external potential, every solution is dispersive, with a universal asymptotic profile. The introduction of a harmonic potential generates solitary waves, corresponding to generalized Gaussons. We prove that they are orbitally stable, using an inequality related to relative entropy, which may be thought of as dual to the classical logarithmic Sobolev inequality. In the case of a partial confinement, we show a universal dispersive behavior for suitable marginals. For repulsive harmonic potentials, the dispersive rate is dictated by the potential, and no universal behavior must be expected.
\end{abstract}

\section{Introduction}

We consider the logarithmic Schrödinger equation in the presence of an external potential,

$$
i \partial_{t} u+\frac{1}{2} \Delta u=V(x) u+\lambda \ln \left(|u|^{2}\right) u, \quad u_{\mid t=0}=u_{0},
$$

with $x \in \mathbb{R}^{d}, d \geqslant 1$, and $\lambda \in \mathbb{R}$. The potential $V$ is smooth and real-valued, $V \in$ $C^{\infty}\left(\mathbb{R}^{d} ; \mathbb{R}\right)$, and the main results of the present paper concern the case where $V$ is quadratic in $x$. This equation is Hamiltonian; mass and energy are formally independent of time:

$$
\begin{aligned}
M(u(t))= & \|u(t)\|_{L^{2}\left(\mathbb{R}^{d}\right)}^{2}, \\
E(u(t)):= & \frac{1}{2}\|\nabla u(t)\|_{L^{2}\left(\mathbb{R}^{d}\right)}^{2}+\int_{\mathbb{R}^{d}} V(x)|u(t, x)|^{2} d x \\
& +\lambda \int_{\mathbb{R}^{d}}|u(t, x)|^{2}\left(\ln |u(t, x)|^{2}-1\right) d x .
\end{aligned}
$$

On the other hand, due to the presence of the potential $V$, the momentum,

$$
\operatorname{Im} \int_{\mathbb{R}^{d}} \bar{u}(t, x) \nabla u(t, x) d x
$$

is not conserved. As it will not be considered in this paper, we do not discuss the evolution of this quantity. The logarithmic nonlinearity was introduced in $[\mathbf{8}]$ to satisfy the following

$\mathrm{RC}$ is supported by Rennes Métropole through its AIS program. 
tensorization property: if $V$ decouples space variables in the sense that

$$
V(x)=\sum_{j=1}^{d} V_{j}\left(x_{j}\right)
$$

where $V_{j} \in C^{\infty}(\mathbb{R} ; \mathbb{R})$, and if the initial datum is a tensor product,

$$
u_{0}(x)=\prod_{j=1}^{d} u_{0 j}\left(x_{j}\right)
$$

then the solution to 1.1 is given by

$$
u(t, x)=\prod_{j=1}^{d} u_{j}\left(t, x_{j}\right),
$$

where each $u_{j}$ solves a one-dimensional equation,

$$
i \partial_{t} u_{j}+\frac{1}{2} \partial_{x_{j}}^{2} u_{j}=V_{j}\left(x_{j}\right) u_{j}+\lambda \ln \left(\left|u_{j}\right|^{2}\right) u_{j}, \quad u_{j \mid t=0}=u_{0 j} .
$$

Of course, a similar property remains if e.g. $V\left(x_{1}, x_{2}, x_{3}\right)=V_{12}\left(x_{1}, x_{2}\right)+V_{3}\left(x_{3}\right)$, and $u_{0}\left(x_{1}, x_{2}, x_{3}\right)=u_{012}\left(x_{1}, x_{2}\right) u_{03}\left(x_{3}\right)$. As can easily be checked, the above logarithmic nonlinearity is the only one satisfying this tensorization property. This property will be evoked several times in the rest of this paper.

The logarithmic nonlinearity has since been adopted in several physical models, in quantum mechanics [37], quantum optics [8, 26, 31, 12], nuclear physics [28], Bohmian mechanics [32], effective quantum gravity [38], theory of superfluidity and Bose-Einstein condensation $[\overline{5}]$. See also $[\mathbf{3 3}, \mathbf{3 4}]$. The papers $[\mathbf{3 8}, \mathbf{3 9}, \mathbf{1 0}]$ have provided evidences that the logarithmic model may generalize the Gross-Pitaevskii equation, used in the case of two-body interaction, to the case of three-body interaction.

As proposed in [38, 39], the logarithmic nonlinearity may appear as a relevant model to provide a universal mechanism describing the deformation of the quantum wave equation due to non-trivial vacuum. It thus appears as a serious candidate to extend quantum mechanics thanks to a nonlinear model, likely to help understand quantum gravity. In the absence of external potential $(V=0)$, the case $\lambda<0$ is certainly the most physically relevant: stationary solutions known as Gaussons are present and stable under the dynamics (see below), while for $\lambda>0$, (enhanced) dispersion is always present; see Theorem 1.1 for a complete mathematical statement, showing that it is indeed hopeless to look for stable structures if $\lambda>0$.

In [10], the presence of an harmonic trap was considered, in order to describe logarithmic BEC. As in the case without potential, an important feature of the model is that many Gaussian solutions are available, as explored into more details in Section 3.3 Stationary solutions (generalized Gaussons) are still available in the case $\lambda<0$. Their stability was investigated numerically in [10], and analyzed mathematically in [4]. The presence of the harmonic trap implies that stable structures are now also present in the case $\lambda>0$, as shown numerically in [10], and analyzed more precisely in the present paper.

Considering for $V$ a harmonic potential is not only physically relevant, it has also a natural mathematical motivation, which we now explain. 
1.1. Mathematical background. The first mathematical study of (1.1) appears in [19] (see also [18]), where the Cauchy problem is addressed for $V \in L^{p}\left(\mathbb{R}^{d}\right)+L^{\infty}\left(\mathbb{R}^{d}\right)$, with $p \geqslant 1$ and $p>d / 2$. As noticed in [9], in the case $\lambda<0$ with $V=0$, (1.1) admits a solitary wave of the form $e^{i \omega t} \phi(x)$, where $\phi$ is a Gaussian, called Gausson. The orbital stability of this solitary wave was established in [17] in the radial case, and in [3] in the general case. Still in the case $\lambda<0$ with $V=0$, it was shown in [19] that if $u_{0} \in W$, the energy space given by

$$
W=\left\{f \in H^{1}\left(\mathbb{R}^{d}\right), \quad|f|^{2} \ln |f|^{2} \in L^{1}\left(\mathbb{R}^{d}\right)\right\},
$$

then there exists a unique, global, solution $u \in L^{\infty}(\mathbb{R} ; W)$ (see also [23, 27]), and in [17] that no such solution is dispersive. The global Cauchy problem in the case $V=0, \lambda>0$ was treated in [23] and [16].

As noticed in [8], in the case $V=0, \lambda \in \mathbb{R}$, if $u_{0}$ is a Gaussian, then $u(t, \cdot)$ is a Gaussian for all time. The evolution of Gaussians was analyzed more precisely in [6, 16, 21]: in the case $\lambda<0$, they behave like breathers, in the sense that $|u(t, \cdot)|$ is periodic in time, while if $\lambda>0$, they all are dispersive with a similar rate $\tau(t)$, and $\tau(t)^{d}|u(t, x \tau(t))|^{2}$ has a universal limit, that is, a Gaussian whose variance does not depend on the initial Gaussian $u_{0}$.

The latter property has been established for initial data which are not necessarily Gaussian, in [16], and the description of the convergence was refined in [22]. Denote

$$
\Sigma=H^{1} \cap \mathcal{F}\left(H^{1}\right)=\left\{f \in H^{1}\left(\mathbb{R}^{d}\right), \quad x \mapsto|x| f(x) \in L^{2}\left(\mathbb{R}^{d}\right)\right\} .
$$

THEOREM 1.1 ([16, 22]). Let $V=0$ and $\lambda>0$. For $u_{0} \in \Sigma \backslash\{0\}$, 1.1] has a unique solution $u \in L_{\mathrm{loc}}^{\infty}(\mathbb{R} ; \Sigma)$ ). Introduce the solution $\tau \in C^{\infty}(\mathbb{R})$ to the ODE

$$
\ddot{\tau}=\frac{2 \lambda}{\tau}, \quad \tau(0)=1, \quad \dot{\tau}(0)=0 .
$$

Then, as $t \rightarrow \infty, \tau(t) \sim 2 t \sqrt{\lambda \ln t}$ and $\dot{\tau}(t) \sim 2 \sqrt{\lambda \ln t}$. Introduce $\gamma(x):=e^{-|x|^{2} / 2}$, and rescale the solution to $v=v(t, y)$ by setting

$$
u(t, x)=\frac{1}{\tau(t)^{d / 2}} v\left(t, \frac{x}{\tau(t)}\right) \frac{\left\|u_{0}\right\|_{L^{2}\left(\mathbb{R}^{d}\right)}}{\|\gamma\|_{L^{2}\left(\mathbb{R}^{d}\right)}} \exp \left(i \frac{\dot{\tau}(t)}{\tau(t)} \frac{|x|^{2}}{2}\right) .
$$

Then we have

$$
\int_{\mathbb{R}^{d}}\left(\begin{array}{c}
1 \\
y \\
|y|^{2}
\end{array}\right)|v(t, y)|^{2} d y \underset{t \rightarrow \infty}{\longrightarrow} \int_{\mathbb{R}^{d}}\left(\begin{array}{c}
1 \\
y \\
|y|^{2}
\end{array}\right) \gamma^{2}(y) d y
$$

and

$$
|v(t, \cdot)|^{2} \underset{t \rightarrow \infty}{\rightarrow} \gamma^{2} \quad \text { weakly in } L^{1}\left(\mathbb{R}^{d}\right) .
$$

Finally, denoting by $W_{1}$ the Wasserstein distance $\rrbracket$ there exists $C$ such that

$$
W_{1}\left(\frac{|v(t)|^{2}}{\pi^{d / 2}}, \frac{\gamma^{2}}{\pi^{d / 2}}\right) \leqslant \frac{C}{\sqrt{\ln t}}, \quad t \geqslant e .
$$

\footnotetext{
${ }^{1}$ For $\nu_{1}$ and $\nu_{2}$ probability measures,

$$
W_{1}\left(\nu_{1}, \nu_{2}\right)=\inf \left\{\int_{\mathbb{R}^{d} \times \mathbb{R}^{d}}|x-y| d \mu(x, y) ; \quad\left(\pi_{j}\right)_{\sharp} \mu=\nu_{j}\right\},
$$

where $\mu$ varies among all probability measures on $\mathbb{R}^{d} \times \mathbb{R}^{d}$, and $\pi_{j}: \mathbb{R}^{d} \times \mathbb{R}^{d} \rightarrow \mathbb{R}^{d}$ denotes the canonical projection onto the $j$-th factor. See e.g. 35
} 
The case where $V$ is harmonic, $V(x)=|x|^{2}$, was considered in [4], in the case $\lambda<0$ : there exists an analogue of the Gausson, that is a solution of the form $b_{0} e^{i \omega t} e^{-a_{0}|x|^{2} / 2}$, and it is orbitally stable. In the following statement, we adapt the numerical values to 1.1 and unify the statements from [3] (without potential) and [4] (with potential, where (1.1) is no longer translation invariant):

THEOREM $1.2([\mathbf{3}, 4])$. Let $d \geqslant 1$ and $\lambda<0$. Suppose that

$$
V(x)=\frac{\kappa(\kappa+2 \lambda)}{2}|x|^{2}, \quad \kappa \geqslant-2 \lambda>0 .
$$

Then the (generalized) Gausson is given by $\phi_{\nu}(x)=e^{-\frac{\nu+\kappa d / 2}{2 \lambda}} e^{-\kappa|x|^{2} / 2}$, for $\nu \in \mathbb{R}$. It generates a standing wave $u(t, x)=\phi_{\nu}(x) e^{\text {ivt }}$ solution to [1.1, which is orbitally stable in the energy space: For any $\varepsilon>0$, there exists $\eta>0$ such that if $u_{0} \in X$ satisfies $\left\|u_{0}-\phi_{\nu}\right\|_{X}<\eta$, then the solution $u$ to (1.1) exists for all $t \in \mathbb{R}$, and

- Case without potential: $\kappa=-2 \lambda$ and $X=W$,

$$
\sup _{t \in \mathbb{R}} \inf _{\theta \in \mathbb{R}} \inf _{y \in \mathbb{R}^{d}}\left\|u(t)-e^{i \theta} \phi_{\nu}(\cdot-y)\right\|_{W}<\varepsilon .
$$

- Case with potential: $\kappa>-2 \lambda$ and $X=\Sigma$,

$$
\sup _{t \in \mathbb{R}} \inf _{\theta \in \mathbb{R}}\left\|u(t)-e^{i \theta} \phi_{\nu}\right\|_{\Sigma}<\varepsilon .
$$

1.2. Main results. To make things clear, we first show that the Cauchy problem 1.1) is globally well-posed in $\Sigma$, provided that the potential $V$ is smooth and at most quadratic. This naturally generalizes the results known in the case of a power nonlinearity (see e.g. [15]).

Proposition 1.3. Let $V \in C^{\infty}\left(\mathbb{R}^{d} ; \mathbb{R}\right)$, at most quadratic, in the sense that $\partial^{\alpha} V \in$ $L^{\infty}\left(\mathbb{R}^{d}\right)$ as soon as $|\alpha| \geqslant 2$. For $u_{0} \in \Sigma$, there exists a unique solution $u \in L_{\mathrm{loc}}^{\infty}(\mathbb{R} ; \Sigma) \cap$ $C\left(\mathbb{R} ; L^{2}\left(\mathbb{R}^{d}\right)\right)$ to 11.1 . Moreover, the mass $M(u(t))$ and the energy $E(u(t))$ are independent of time.

The above result was established in [4] in the case where $V$ is an isotropic harmonic potential, and $\lambda<0$. We prove Proposition 1.3 by adapting the strategy from [16], which is different from the one in [4], inspired by [19].

REMARK 1.4. The case of a time dependent potential $V$ could be considered as well, with very few modifications regarding Proposition 1.3] (see [15]), as well as in the description of the propagation of Gaussian functions in Section 3.3 .

In the rest of this introduction, and for the other results, we assume $\lambda>0$.

1.2.1. Full harmonic confinement. We first consider the case of a full, isotropic confinement: $V(x)=\frac{\omega^{2}}{2}|x|^{2}$. This confinement completely alters the dynamics of the case $\lambda>0$, since solitary waves now exist, while from Theorem 1.1, all $\Sigma$-solutions are dispersive when $V=0$. To make the connexion with the case $\lambda<0$ considered in [4] explicit, rewrite $\omega^{2}$ as $\omega^{2}=\kappa(\kappa+2 \lambda), \kappa>0$.

THEOREM 1.5. Let $d \geqslant 1$ and $\lambda>0$. Suppose that

$$
V(x)=\frac{\kappa(\kappa+2 \lambda)}{2}|x|^{2}, \quad \kappa>0 .
$$

Then the (generalized) Gausson is given by $\phi_{\nu}(x)=e^{-\frac{\nu+\kappa d / 2}{2 \lambda}} e^{-\kappa|x|^{2} / 2}$, for $\nu \in \mathbb{R}$. It generates a standing wave $u(t, x)=\phi_{\nu}(x) e^{\text {ivt }}$ solution to $1 \mathbf{1 . 1}$, which is orbitally stable 
in the energy space: For any $\varepsilon>0$, there exists $\eta>0$ such that if $u_{0} \in \Sigma$ satisfies $\left\|u_{0}-\phi_{\nu}\right\|_{\Sigma}<\eta$, then the solution $u$ to (1.1) exists for all $t \in \mathbb{R}$, and

$$
\sup _{t \in \mathbb{R}} \inf _{\theta \in \mathbb{R}}\left\|u(t)-e^{i \theta} \phi_{\nu}\right\|_{\Sigma}<\varepsilon .
$$

We see in particular that in the limit case $V=0$, corresponding to $\kappa=0, \phi_{\nu}$ is no longer an $L^{2}$-function. To prove Theorem 1.5 we essentially resume the approach from [4], based on the Cazenave-Lions method [20], as well as on a variational characterization of the generalized Gausson. In the case $\lambda<0$, this characterization relies on the logarithmic Sobolev inequality and the description of equality cases. In the present framework, the logarithmic Sobolev inequality is replaced by a rather natural counterpart, involving a momentum instead of a derivative, see Lemma4.2

REMARK 1.6. The case of anisotropic confinement in all directions,

$$
V(x)=\sum_{j=1}^{d} \frac{\omega_{j}^{2}}{2} x_{j}^{2}, \quad \omega_{j}>0,
$$

can be addressed with straightforward adaptations, by considering suitable anisotropic Gaussian functions in all steps of the proof, including Lemma 4.2

1.2.2. Partial harmonic confinement. In the same spirit as e.g. [2, 7], we now assume $d \geqslant 2$, and that $V$ is confining in some but not all directions: suppose that the space variable is now $\left(x^{\prime}, x^{\prime \prime}\right) \in \mathbb{R}^{d_{1}} \times \mathbb{R}^{d_{2}}$, with $d_{1}, d_{2} \geqslant 1, d_{1}+d_{2}=d$, and

$$
V\left(x^{\prime}, x^{\prime \prime}\right)=\frac{\omega^{2}}{2}\left|x^{\prime}\right|^{2} .
$$

We show that dispersion is always present in $x^{\prime \prime}$, in the sense that Theorem 1.1 remains valid for $u$, provided that suitable integration in $x^{\prime}$ is considered.

TheOREM 1.7. Let $\left(x^{\prime}, x^{\prime \prime}\right) \in \mathbb{R}^{d_{1}} \times \mathbb{R}^{d_{2}}$, with $d_{1}, d_{2} \geqslant 1, d_{1}+d_{2}=d$, and

$$
V\left(x^{\prime}, x^{\prime \prime}\right)=\frac{\omega^{2}}{2}\left|x^{\prime}\right|^{2}, \quad \omega>0 .
$$

Suppose $\lambda>0$. Let $u_{0} \in \Sigma \backslash\{0\}$, and resume the notation $\gamma\left(x^{\prime \prime}\right):=e^{-\left|x^{\prime \prime}\right|^{2} / 2}$. Introduce

$$
\rho(t, y):=\tau(t)^{d_{2}} \int_{\mathbb{R}^{d_{1}}}\left|u\left(t, x^{\prime}, y \tau(t)\right)\right|^{2} d x^{\prime} \times \frac{\pi^{d_{2}}}{\left\|u_{0}\right\|_{L^{2}\left(\mathbb{R}^{d}\right)}^{2}} .
$$

Then we have

and

$$
\int_{\mathbb{R}^{d_{2}}}\left(\begin{array}{c}
1 \\
y \\
|y|^{2}
\end{array}\right) \rho(t, y) d y \underset{t \rightarrow \infty}{\longrightarrow} \int_{\mathbb{R}^{d_{2}}}\left(\begin{array}{c}
1 \\
y \\
|y|^{2}
\end{array}\right) \gamma^{2}(y) d y
$$

along with

$$
\rho(t, \cdot) \underset{t \rightarrow \infty}{\rightarrow} \gamma^{2} \quad \text { weakly in } L^{1}\left(\mathbb{R}^{d}\right)
$$

$$
W_{1}\left(\frac{|\rho(t)|^{2}}{\pi^{d_{2} / 2}}, \frac{\gamma^{2}}{\pi^{d_{2} / 2}}\right) \leqslant \frac{C}{\sqrt{\ln t}}, \quad t \geqslant e .
$$

Theorem 1.7 can be informally restated by saying that

$$
\int_{\mathbb{R}^{d_{1}}}\left|u\left(t, x^{\prime}, x^{\prime \prime}\right)\right|^{2} d x^{\prime}
$$


is dispersive in the variable $x^{\prime \prime}$, with rate $\tau(t)^{-d_{2}}$. Such a dispersive property on a marginal was used in [2] as a first step (following from Morawetz estimates) to prove scattering for NLS with a power-like nonlinearity and a partial confinement. In the present framework, no scattering result can be inferred.

Indeed, considering tensorized initial data of the form $u_{0}(x)=u_{01}\left(x^{\prime}\right) u_{02}\left(x^{\prime \prime}\right)$, we have $u(t, x)=u_{1}\left(t, x^{\prime}\right) u_{2}\left(t, x^{\prime \prime}\right)$, where $u_{1}$ solves (1.1) with a fully confining potential, and $u_{2}$ solves 1.1 with $V=0$, hence obeys Theorem 1.1 (with $d$ replaced by $d_{2}$ ). In particular, $u_{1}$ may correspond to solutions described in Theorem 1.5, or be a Gaussian breather (see Section 3.3), showing that integrating with respect to $x^{\prime}$ first, in the above result, makes perfect sense, and the dynamics in the $x^{\prime}$ variable is independent of the dispersion stated in Theorem 1.7

1.2.3. Repulsive harmonic potential. We finally present some results in the repulsive harmonic case,

$$
i \partial_{t} u+\frac{1}{2} \Delta u=-\omega^{2} \frac{|x|^{2}}{2} u+\lambda u \ln \left(|u|^{2}\right), \quad u_{\mid t=0}=u_{0},
$$

for $x \in \mathbb{R}^{d}$ and $\omega, \lambda>0$. In the case $\lambda=0$, any defocusing energy-subcritical power-like nonlinearity $|u|^{2 \sigma} u, 0<\sigma<\frac{2}{(d-2)_{+}}$, is short range for scattering, since the repulsive harmonic potential induces an exponential decay in time ([13]). In particular, in the dispersive frame (meaning after rescaling the wave function in terms of the time dependent dispersion, $e^{\omega t}$ in this case), any asymptotic profile can be reached. On the other hand for $\omega=0$, Theorem 1.1 shows that there is only one profile (if one considers the modulus only) which can be reached in the dispersive frame. The case $\omega, \lambda>0$ is therefore a borderline case from these two perspectives: do we have scattering, or a universal behavior? In the next result, we give a partial answer to this question, showing the same dispersive rate as in the linear case $\lambda=0$, and ruling out a universal behavior in the sense of Theorem 1.1

Proposition 1.8. Let $\omega, \lambda>0$ and let $\tau_{-}$be the solution to the $O D E$

$$
\ddot{\tau}_{-}=\omega^{2} \tau_{-}+\frac{2 \lambda}{\tau_{-}}, \quad \tau_{-}(0)=1, \quad \dot{\tau}_{-}(0)=0 .
$$

There exists $\mu_{\infty}>0$ such that, as $t \rightarrow \infty, \tau_{-}(t) \sim \mu_{\infty} e^{\omega t}, \dot{\tau}_{-}(t) \sim \omega \mu_{\infty} e^{\omega t}$. For $u_{0} \in \Sigma$ and the solution $u \in L_{\mathrm{loc}}^{\infty}(\mathbb{R} ; \Sigma)$ to $[1.5]$, consider the rescaling

$$
u(t, x)=\frac{1}{\tau_{-}(t)^{d / 2}} v\left(t, \frac{x}{\tau_{-}(t)}\right) \exp \left(i \frac{\dot{\tau}_{-}(t)}{\tau_{-}(t)} \frac{|x|^{2}}{2}\right) .
$$

Then $v$ is bounded and not dispersive, in the sense that

$$
\sup _{t \geqslant 0} \int_{\mathbb{R}^{d}}\left(1+|y|^{2}+\left.|\ln | v(t, y)\right|^{2} \mid\right)|v(t, y)|^{2} d y<\infty .
$$

We can find two initial data

$$
u_{01}(x)=e^{-|x|^{2} / 2+i \beta_{1}|x|^{2} / 2}, \quad u_{02}(x)=e^{-|x|^{2} / 2+i \beta_{2}|x|^{2} / 2}, \quad \beta_{j}>0,
$$

such that the corresponding $v_{j}$ 's satisfy

$$
\left|v_{j}(t, \cdot)\right|^{2} \underset{t \rightarrow \infty}{\longrightarrow} \gamma_{j}^{2} \text { in } L^{1}\left(\mathbb{R}^{d}\right), \quad \text { with } \gamma_{1} \neq \gamma_{2} .
$$

In the same spirit as the comments following the statement of Theorem 1.7 we emphasize that the dynamics associated to the logarithmic nonlinearity 1.1 is completely 
different from the dynamics for NLS with a power-type nonlinearity. Typically, in [14], the case of a saddle potential, for $x \in \mathbb{R}^{d}, d \geqslant 2$,

$$
V(x)=\omega_{1}^{2} x_{1}^{2}-\omega_{2}^{2} x_{2}^{2},
$$

was considered. There is confinement in the first direction, and a strong dispersion in the second one. In the case of a defocusing power-like nonlinearity $|u|^{2 \sigma} u$, we have global existence in $\Sigma$. Given $u_{0} \in \Sigma$, at least if $\sigma \geqslant 2 / d$ and $\omega_{2}$ is sufficiently large compared to $\omega_{1}$, there is scattering in the sense that there exist $u_{ \pm} \in \Sigma$ such that

$$
\left\|U_{V}(-t) u(t)-u_{ \pm}\right\|_{\Sigma} \underset{t \rightarrow \pm \infty}{\longrightarrow} 0, \quad U_{V}(t)=e^{-i t H}, \quad H=-\frac{1}{2} \Delta+V .
$$

Proposition 1.8 shows that for a logarithmic nonlinearity, there is still an exponential dispersion in $x_{2}$, but the tensorization property implies that no scattering result can hold.

1.3. Outline of the paper. In Section 2, we show how to prove Proposition 1.3, In Section 3, we describe more precisely some remarkable properties related to the logarithmic nonlinearity in the presence of a quadratic potential: invariances, special transforms, and propagation of Gaussian data. Theorem 1.5 is proven in Section 4 relying on Lemma 4.2 which can be thought of as a dual to the logarithmic Sobolev inequality. In Section 5 we address the proof of Theorem 1.7, by showing essentially how to consider a suitable setting in order to relate this result to Theorem 1.1 Finally, Proposition 1.8 is proved in Section 6

\section{Cauchy problem}

In this section, we briefly explain the proof of Proposition 1.3 , which can be addressed like in the case without potential considered in [16]. The main issue is that the logarithmic nonlinearity is not Lipschitz continuous at the origin. Proceeding as in [16], we first regularize the nonlinearity by saturating the logarithm near zero, and consider the sequence of approximate solutions given by

$$
i \partial_{t} u^{\varepsilon}+\frac{1}{2} \Delta u^{\varepsilon}=V(x) u^{\varepsilon}+\lambda \ln \left(\varepsilon+\left|u^{\varepsilon}\right|^{2}\right) u^{\varepsilon}, \quad u_{\mid t=0}^{\varepsilon}=u_{0},
$$

where $V, \lambda$ and $u_{0}$ are as in Proposition 1.3, and $\varepsilon>0$. For fixed $\varepsilon>0$, the above nonlinearity is locally Lipschitzian, with moderate growth at infinity, ensuring that it is $L^{2}$ subcritical. Since the potential $V$ is smooth and at most quadratic, local-in-time Strichartz estimates are available for $H=-\frac{1}{2} \Delta+V$, the same as in the case $V=0$ (see e.g. [15] and references therein). Therefore, for every $\varepsilon>0$, there is a unique, global solution at the $L^{2}$ level. Again, at fixed $\varepsilon>0$, the nonlinearity is smooth, so higher regularity is propagated: $u^{\varepsilon}, \nabla u^{\varepsilon}, x u^{\varepsilon} \in C\left(\mathbb{R} ; L^{2}\left(\mathbb{R}^{d}\right)\right)$.

The sequence $\left(u^{\varepsilon}\right)_{0<\varepsilon \leqslant 1}$ converges, thanks to compactness arguments based on uniform a priori estimates. Since $\lambda \in \mathbb{R}$ and $V$ is real-valued, the $L^{2}$-norm of $u^{\varepsilon}$ is independent of time, $\left\|u^{\varepsilon}(t)\right\|_{L^{2}}=\left\|u_{0}\right\|_{L^{2}}$. For $1 \leqslant j \leqslant d$, differentiating (2.1) with respect to $x_{j}$ yields

$$
\begin{aligned}
i \partial_{t} \partial_{j} u_{\varepsilon}+\frac{1}{2} \Delta \partial_{j} u_{\varepsilon}= & V(x) \partial_{j} u^{\varepsilon}+\partial_{j} V(x) u^{\varepsilon}+\lambda \ln \left(\varepsilon+\left|u_{\varepsilon}\right|^{2}\right) \partial_{j} u_{\varepsilon} \\
& +2 \lambda \frac{1}{\varepsilon+\left|u_{\varepsilon}\right|^{2}} \operatorname{Re}\left(\bar{u}_{\varepsilon} \partial_{j} u_{\varepsilon}\right) u_{\varepsilon} .
\end{aligned}
$$


By assumption on $V,\left|\partial_{j} V(x)\right| \lesssim 1+|x|$, so the standard $L^{2}$ estimate yields

$$
\frac{d}{d t}\left\|\nabla u^{\varepsilon}(t)\right\|_{L^{2}}^{2} \leqslant C\left(\left\|u^{\varepsilon}(t)\right\|_{L^{2}}^{2}+\left\|x u^{\varepsilon}(t)\right\|_{L^{2}}^{2}+\left\|\nabla u^{\varepsilon}(t)\right\|_{L^{2}}^{2}\right),
$$

where $C$ is independent of $\varepsilon$. Similarly,

$$
i \partial_{t}\left(x_{j} u_{\varepsilon}\right)+\frac{1}{2} \Delta\left(x_{j} u_{\varepsilon}\right)=\partial_{j} u^{\varepsilon}+V(x) x_{j} u^{\varepsilon}+\lambda \ln \left(\varepsilon+\left|u_{\varepsilon}\right|^{2}\right) x_{j} u_{\varepsilon},
$$

hence

$$
\frac{d}{d t}\left\|x u^{\varepsilon}(t)\right\|_{L^{2}}^{2} \leqslant 2 \int_{\mathbb{R}^{d}}\left|x u^{\varepsilon}(t, x)\left\|\nabla u^{\varepsilon}(t, x) \mid d x \leqslant\right\| x u^{\varepsilon}(t)\left\|_{L^{2}}^{2}+\right\| \nabla u^{\varepsilon}(t) \|_{L^{2}}^{2} .\right.
$$

In view of the conservation of the mass, Gronwall lemma implies that there exists $C$ independent of $\varepsilon$ such that

$$
\left\|x u^{\varepsilon}(t)\right\|_{L^{2}}^{2}+\left\|\nabla u^{\varepsilon}(t)\right\|_{L^{2}}^{2} \leqslant C\left(\left\|u_{0}\right\|_{L^{2}}^{2}+\left\|x u_{0}\right\|_{L^{2}}^{2}+\left\|\nabla u_{0}\right\|_{L^{2}}^{2}\right) e^{C|t|}, \quad t \in \mathbb{R} .
$$

Therefore, we have compactness in space for the sequence $\left(u^{\varepsilon}\right)_{\varepsilon}$. Compactness in time follows from 2.11. Arzela-Ascoli theorem yields a converging subsequence, hence the existence part of Proposition 1.3 .

The conservation of mass and energy can be proven like in [19] (see also [18]).

Uniqueness follows from the remark that any solution $u \in L_{\text {loc }}^{\infty}(\mathbb{R} ; \Sigma)$ to 1.1 actually belongs to $C\left(\mathbb{R} ; L^{2}\left(\mathbb{R}^{d}\right)\right.$ ), since the equation implies $\partial_{t} u \in L_{\text {loc }}^{\infty}\left(\mathbb{R} ; H^{-1}+\mathcal{F}\left(H^{-1}\right)\right)$ (see [16]), and from the argument discovered in [19]:

LEMMa 2.1 (Lemma 9.3.5 from [18]). We have

$$
\left|\operatorname{Im}\left(\left(z_{2} \ln \left|z_{2}\right|^{2}-z_{1} \ln \left|z_{1}\right|^{2}\right)\left(\bar{z}_{2}-\bar{z}_{1}\right)\right)\right| \leqslant 4\left|z_{2}-z_{1}\right|^{2}, \quad \forall z_{1}, z_{2} \in \mathbb{C} .
$$

For two solutions $u_{1}, u_{2} \in L_{\text {loc }}^{\infty}(\mathbb{R} ; \Sigma)$ to 1.1 , the difference $w=u_{2}-u_{1}$ solves

$$
i \partial_{t} w+\frac{1}{2} \Delta w=V(x) w+\lambda\left(\ln \left(\left|u_{2}\right|^{2}\right) u_{2}-\ln \left(\left|u_{1}\right|^{2}\right) u_{1}\right), \quad w_{\mid t=0}=0,
$$

and the standard $L^{2}$ estimate yields, along with the above lemma,

$$
\begin{aligned}
\frac{1}{2} \frac{d}{d t}\|w(t)\|_{L^{2}\left(\mathbb{R}^{d}\right)}^{2} & =\lambda \operatorname{Im} \int_{\mathbb{R}^{d}}\left(\ln \left(\left|u_{2}\right|^{2}\right) u_{2}-\ln \left(\left|u_{1}\right|^{2}\right) u_{1}\right)\left(\bar{u}_{2}-\bar{u}_{1}\right)(t) d x \\
& \leqslant 4 \lambda\|w(t)\|_{L^{2}\left(\mathbb{R}^{d}\right)}^{2},
\end{aligned}
$$

hence $w \equiv 0$ from Gronwall lemma.

\section{Remarkable algebraic properties of 1.1p}

3.1. Invariants. Apart from the conservations of mass and energy, 1.11 is invariant with respect to translation in time, but not in space in general, due to the potential $V$. When $V$ is zero or exactly quadratic, there is a Galilean invariance (whose expression depends on the signature of $\nabla^{2} V$ ). Having the tensorization property in mind, recall the formulas for $d=1$ :

- Case $V=0$ : if $u(t, x)$ solve (1.1), then for any $v \in \mathbb{R}$, so does $u(t, x-$ $v t) e^{i v x-i v^{2} t / 2}$.

- Case $V(x)=\omega^{2} x^{2} / 2$ : if $u(t, x)$ solve 1.11, then for any $v \in \mathbb{R}$, so does

$$
u\left(t, x-v \frac{\sin (\omega t)}{\omega}\right) e^{i v x \cos (\omega t)-i v^{2} \cos (\omega t) \frac{\sin (\omega t)}{2 \omega}} .
$$


- Case $V(x)=-\omega^{2} x^{2} / 2$ : if $u(t, x)$ solve 11.1 , then for any $v \in \mathbb{R}$, so does

$$
u\left(t, x-v \frac{\sinh (\omega t)}{\omega}\right) e^{i v x \cosh (\omega t)-i v^{2} \cosh (\omega t) \frac{\sinh (\omega t)}{2 \omega}} .
$$

The logarithmic nonlinearity causes a rather unique invariance, as noticed in [16] for $V=$ 0 , a property which remains in the presence of $V$ : If $u$ solves 1.1 , then for all $k \in \mathbb{C}$, so does

$$
k u(t, x) e^{-i t \lambda \ln |k|^{2}} .
$$

This shows that the size of the initial data alters the dynamics only through a purely time dependent oscillation, a feature which is fairly unusual for a nonlinear equation. In addition, considering $k>0$, differentiating the above formula with respect to $k$ and letting $k \rightarrow 0$ shows that for $t>0$ arbitrarily small, the flow map $u_{0} \mapsto u(t)$ cannot be $C^{1}$, whichever function spaces are considered for $u_{0}$ and $u(t)$, respectively; it is at most Lipschitzian.

3.2. Special potentials. It is a standard fact that if $V$ is linear in $x, V(x)=E \cdot x$ for some fixed $E \in \mathbb{R}^{d}$, the influence of $V$ is explicit, in the sense that if $u$ solves (1.1) with this $V$, and $v$ solves (1.1) with $V=0$ (and the same initial datum), then $u$ and $v$ are related through

$$
v(t, x)=u\left(t, x-\frac{t^{2}}{2} E\right) e^{i\left(t E \cdot x-\frac{t^{3}}{3}|E|^{2}\right)} .
$$

See e.g. [15], where this formula is extended to the case where $E$ depends on time.

On the other hand, lens transforms (see e.g. [15]) seem to be useless in the case of a logarithmic nonlinearity. If $u$ solves (1.1) with $V=0$, for $\omega>0$ and $|t|<\pi /(2 \omega)$, set

$$
w^{+}(t, x)=\frac{1}{(\cos (\omega t))^{d / 2}} e^{-i \frac{\omega}{2}|x|^{2} \tan (\omega t)} u\left(\frac{\tan (\omega t)}{\omega}, \frac{x}{\cos (\omega t)}\right) .
$$

Then $w^{+}$solves

$$
i \partial_{t} w^{+}+\frac{1}{2} \Delta w^{+}=\frac{\lambda}{\cos (\omega t)^{2}} \ln \left(\cos (\omega t)^{d}\left|w^{+}\right|^{2}\right) w^{+}+\omega^{2} \frac{|x|^{2}}{2} w^{+}, \quad w_{\mid t=0}^{+}=u_{0} .
$$

The time dependent factor in front of the nonlinearity shows a strong difference with 1.11, and the equation in $w^{+}$is not necessarily more pleasant to study. The case of a repulsive harmonic potential $V(x)=-\omega^{2}|x|^{2} / 2$ is similar (replace $\omega$ by $i \omega$ ).

3.3. Propagation of Gaussian initial data. A remarkable feature of 1.1 is that when the potential is a polynomial of degree at most two in space, then an initial Gaussian data evolves as a Gaussian for all time. Plugging a time-dependent Gaussian function into the logarithmic nonlinearity, we readily see that this remarkable property is related to the well-known fact that the same holds in the case of the linear Schrödinger equation with potential; see e.g. [24, 25, 29, 30].

In view of the tensorization property described in the introduction, we consider the case $d=1$, and a quadratic potential,

$$
i \partial_{t} u+\frac{1}{2} \partial_{x}^{2} u=\lambda \ln \left(|u|^{2}\right) u+\Omega \frac{x^{2}}{2} u, \quad u_{\mid t=0}=u_{0},
$$

where $\Omega \in \mathbb{R}$ is a constant. We seek $u(t, x)=b(t) e^{-a(t) x^{2} / 2}$ (in particular $u_{0}$ is Gaussian). Plugging this into (3.2), we find:

$$
i \dot{b}=\frac{1}{2} a b+\lambda b \ln |b|^{2} \quad ; \quad i \dot{a}=a^{2}+2 \lambda \operatorname{Re} a-\Omega .
$$


Seeking $a$ under the form

$$
a=\frac{1}{\tau^{2}}-i \frac{\dot{\tau}}{\tau}
$$

leads to

$$
\ddot{\tau}=\frac{2 \lambda}{\tau}+\frac{1}{\tau^{3}}-\Omega \tau .
$$

As long as the solution is smooth, multiply by $\dot{\tau}$ and integrate in time:

$$
(\dot{\tau})^{2}=C_{0}+4 \lambda \ln \tau-\frac{1}{\tau^{2}}-\Omega \tau^{2} .
$$

In the case of a (constant) harmonic potential, we write $\Omega=\omega^{2}: \tau$ is bounded, regardless of the sign of $\lambda$. Typically, we have generalized Gaussons for each sign of $\lambda: u(t, x)=$ $C_{\nu} e^{-k x^{2} / 2+i \nu t}$ solves (3.2) if and only if

$$
\omega^{2}-2 \lambda k=k^{2} \quad ; \quad-\nu-\frac{k}{2}=\lambda \ln \left(C_{\nu}^{2}\right) .
$$

For $u$ to be an $L^{2}$ function in space, only one choice is possible for $k$,

$$
k=-\lambda+\sqrt{\lambda^{2}+\omega^{2}} .
$$

Like in [21], we see that $\tau$ is always periodic, and Gaussian data propagate as breathers in general, as a solitary wave in the specific case discussed above. Writing $\omega^{2}=\kappa(\kappa+2 \lambda)$, $\kappa>\max (0,-2 \lambda)$, we recover the generalized Gausson of the introduction, $\phi_{\nu}(x)=$ $e^{-\frac{\nu+\kappa / 2}{2 \lambda}} e^{-\kappa|x|^{2} / 2}$, with $k=\kappa$ (and $d=1$ here).

In the case of a (constant) repulsive harmonic potential, we write $\Omega=-\omega^{2}$, and 3.3 becomes

$$
\ddot{\tau}=\omega^{2} \tau+\frac{2 \lambda}{\tau}+\frac{1}{\tau^{3}} .
$$

Assuming $\tau(0)>0$, we see that $\tau$ remains positive and bounded away from zero for all time, since (3.4) becomes

$$
\dot{\tau}(t)^{2}=C_{0}+\omega^{2} \tau(t)^{2}+4 \lambda \ln \tau(t)-\frac{1}{\tau(t)^{2}} .
$$

Therefore, in the case $\lambda>0$, there exists $\delta>0$ such that

$$
\tau(t) \geqslant \delta, \quad \forall t \in \mathbb{R} .
$$

We infer trivially

$$
\ddot{\tau} \geqslant \omega^{2} \delta
$$

hence

$$
\tau(t) \underset{t \rightarrow \infty}{\longrightarrow} \infty, \quad \dot{\tau}(t) \underset{t \rightarrow \infty}{\longrightarrow} \infty .
$$

Therefore, we may approximate $\tau$ by the solution to

$$
\ddot{\tau}=\omega^{2} \tau .
$$

In particular, the presence of the repulsive harmonic potential causes dispersion with an exponential dispersive rate. We analyze more precisely this situation in Section 6 . 


\section{Full harmonic confinement}

In this section, we prove Theorem 1.5, by following the strategy of Cazenave and Lions [20], and proving some rigidity property of the generalized Gausson. We recall that

$$
V(x)=\frac{\kappa(\kappa+2 \lambda)}{2}|x|^{2}, \quad \kappa>0,
$$

and we denote $\omega^{2}=\kappa(\kappa+2 \lambda)$ for conciseness.

4.1. Technical preliminary. We introduce the action and the Nehari functional:

$$
\begin{aligned}
& S_{\nu}(u):=E(u)+\nu\|u\|_{L^{2}}^{2}, \\
& I_{\nu}(u):=\|\nabla u\|_{L^{2}}^{2}+\omega^{2}\|x u\|_{L^{2}}^{2}+2 \lambda \int_{\mathbb{R}^{d}}|u|^{2} \ln |u|^{2} d x+2 \nu\|u\|_{L^{2}}^{2}=2 S_{\nu}(u)+2 \lambda\|u\|_{L^{2}}^{2} .
\end{aligned}
$$

We also define the quantity

$$
\begin{aligned}
D(\nu) & =\inf \left\{S_{\nu}(u) \mid u \in \Sigma\left(\mathbb{R}^{d}\right) \backslash\{0\}, I_{\nu}(u)=0\right\} \\
& =-\lambda \sup \left\{\|u\|_{L^{2}}^{2} \mid u \in \Sigma\left(\mathbb{R}^{d}\right) \backslash\{0\}, I_{\nu}(u)=0\right\},
\end{aligned}
$$

and the set of ground states by

$$
\mathcal{G}_{\nu}:=\left\{\phi \in \Sigma\left(\mathbb{R}^{d}\right) \backslash\{0\} \mid I_{\nu}(u)=0, S_{\nu}(\phi)=D(\nu)\right\} .
$$

At this stage, we emphasize a major difference between the case of a power nonlinearity and the logarithmic nonlinearity. For power-type nonlinearities, it is standard to either minimize the action, or minimize the energy with a fixed mass, the two approaches being equivalent in the case of homogeneous nonlinearities. In the case of a logarithmic nonlinearity, the Nehari functional is constant along the flow of the equation, and minimizing the action without constraint, or the energy with a fixed mass, does not seem adequate. This characterization of the ground state in the case of a logarithmic nonlinearity is already present in [3, 4]. A key step of the analysis consists in showing that $\mathcal{G}_{\nu}=\left\{e^{i \theta} \phi_{\nu}, \theta \in \mathbb{R}\right\}$, with $\phi_{\nu}$ defined in Theorem 1.5 ,

$$
\phi_{\nu}(x)=e^{-\frac{\nu+\kappa d / 2}{2 \lambda}} e^{-\kappa|x|^{2} / 2} .
$$

First, we note that the energy functional $E$ defined in 1.2 is of class $C^{1}$, and for $u \in \Sigma$, its Fréchet derivative is given by

$$
E^{\prime}(u)=-\Delta u+\omega^{2}|x|^{2} u+\lambda u \ln |u|^{2} .
$$

As a consequence, $S_{\nu}$ and $I_{\nu}$ are of class $C^{1}$, and for $u \in \Sigma$

$$
\left\langle S_{\nu}^{\prime}(u), u\right\rangle=I_{\nu}(u) .
$$

We will also need the following compactness result:

LEMma 4.1. For any sequence $\left(u_{m}\right)$ uniformly bounded in $\Sigma$, there exists a subsequence (still denoted $\left(u_{m}\right)$ ) and some $u \in \Sigma$ such that:

- $u_{m} \underset{m \rightarrow \infty}{\longrightarrow} u$ in $L^{2}\left(\mathbb{R}^{d}\right)$,

- $u_{m} \underset{m \rightarrow \infty}{\longrightarrow}$ ua.e. in $\mathbb{R}^{d}$,

- The following convergence also holds:

$$
\lim _{m \rightarrow \infty} \int_{\mathbb{R}^{d}}\left|u_{m}\right|^{2} \ln \left|u_{m}\right|^{2} d x=\int_{\mathbb{R}^{d}}|u|^{2} \ln |u|^{2} d x .
$$


SKETCH OF THE PROOF. The first two points are standard. For the third point, using the fact that for all $\delta>0$, there exists $C_{\delta}>0$ such that for all $y \geqslant 0$

$$
\left|y^{2} \ln y^{2}\right| \leqslant C_{\delta}\left(y^{2-\delta}+y^{2+\delta}\right)
$$

we get,

$$
\left|\int_{\mathbb{R}^{d}}\right| u_{m}-\left.u\right|^{2} \ln \left|u_{m}-u\right|^{2} d x \mid \leqslant C\left(1+\left\|u_{m}\right\|_{\Sigma}^{2}+\|u\|_{\Sigma}^{2}\right)\left\|u_{m}-u\right\|_{L^{2}} \underset{m \rightarrow \infty}{\longrightarrow} 0 .
$$

The third point then follows from Brézis-Lieb lemma [11], in the convex case (see also [3, Lemma 2.3]).

4.2. Variational analysis. The main novelty to prove orbital stability in the case $\lambda>0$ is a result which may be viewed as a dual of the celebrated logarithmic Sobolev inequality:

Lemma 4.2. Let $f \in \mathcal{F}\left(H^{1}\left(\mathbb{R}^{d}\right)\right)$ and $a>0$ :

$$
-\int_{\mathbb{R}^{d}}|f(x)|^{2} \ln \left(\frac{|f(x)|^{2}}{\|f\|_{L^{2}}^{2}}\right) d x \leqslant a \int_{\mathbb{R}^{d}}|x|^{2}|f(x)|^{2} d x+\frac{d}{2}\|f\|_{L^{2}}^{2} \ln \frac{\pi}{a} .
$$

There is equality if and only if $|f(x)|=c e^{-a|x|^{2} / 2}$, with $c=\|f\|_{L^{2}}(a / \pi)^{d / 2}$. Assuming $\|f\|_{L^{2}}=1$, we also have

$$
-\int_{\mathbb{R}^{d}}|f(x)|^{2} \ln \left(|f(x)|^{2}\right) d x \leqslant \frac{d}{2} \ln \left(\frac{2 e \pi}{d} \int_{\mathbb{R}^{d}}|x|^{2}|f(x)|^{2} d x\right) .
$$

REMARK 4.3. The above inequalities are not translation invariant, just like the usual logarithmic Sobolev inequality is not translation invariant on the Fourier side. However, we must take much more than translation on the Fourier side into account, as the inequality is obviously invariant by multiplication by $e^{i \phi(x)}$ for $\phi$ real-valued, not necessarily linear in $x$.

The second inequality is the counterpart of the classical optimal logarithmic Sobolev inequality, for $\|f\|_{L^{2}}=1$,

$$
\int_{\mathbb{R}^{d}}|f(x)|^{2} \ln \left(|f(x)|^{2}\right) d x \leqslant \frac{d}{2} \ln \left(\frac{d}{2 e \pi}\|\nabla f\|_{L^{2}}^{2}\right),
$$

established in [36].

Proof. For $a>0$, consider the normalized Gaussian

$$
\nu_{a}(x)=\left(\frac{a}{\pi}\right)^{d / 2} e^{-a|x|^{2}} .
$$

It is normalized to ensure that $\nu_{a}$ is a probability density. In view of the Csiszár-Kullback inequality (see e.g. [1 Th. 8.2.7]), for $\mu, \nu$ probability densities,

$$
\|\mu-\nu\|_{L^{1}\left(\mathbb{R}^{d}\right)}^{2} \leqslant 2 \int_{\mathbb{R}^{d}} \mu(x) \ln \left(\frac{\mu(x)}{\nu(x)}\right) d x .
$$

Let $\mu(x)=|f(x)|^{2} /\|f\|_{L^{2}}^{2}$ and $\nu=\nu_{a}$ : since the above relative entropy (right-hand side) is non-negative, we have

$$
\int_{\mathbb{R}^{d}}|f(x)|^{2} \ln \left(\frac{|f(x)|^{2}}{\|f\|_{L^{2}}^{2} \nu_{a}(x)}\right) d x \geqslant 0 .
$$

Replacing $\nu_{a}$ by its explicit value and expanding yields 4.17. 
Moreover, equality holds in (4.1) if and only if the relative entropy is zero, and CsiszárKullback inequality implies that $\mu(x)=\nu_{a}(x)$.

Suppose $\|f\|_{L^{2}}=1$. The map $a \mapsto \alpha a-\beta \ln a+c, \alpha, \beta>0$ reaches its minimum for $a=\beta / \alpha$, so we optimize (4.1) with $\alpha=\|x f\|_{L^{2}}^{2}, \beta=\frac{d}{2}$ and $c=\frac{d}{2} \ln \pi$,

$$
-\int_{\mathbb{R}^{d}}|f(x)|^{2} \ln \left(|f(x)|^{2}\right) d x \leqslant \frac{d}{2} \ln \left(\frac{2 e}{d}\|x f\|_{L^{2}}^{2}\right)+\frac{d}{2} \ln \pi,
$$

hence the announced inequality.

Lemma 4.4 (Ground energy and ground states). Let $\nu \in \mathbb{R}$. Then the quantity $D(\nu)$ is given by

Moreover,

$$
D(\nu)=-\lambda\left\|\phi_{\nu}\right\|_{L^{2}}^{2}=-\lambda \pi^{\frac{d}{2}} \kappa^{-\frac{d}{2}} e^{-\frac{\nu+\kappa d / 2}{\lambda}} .
$$

$$
\mathcal{G}_{\nu}=\left\{e^{i \theta} \phi_{\nu}, \theta \in[0,2 \pi[\} .\right.
$$

Proof. Let $u \in \Sigma \backslash\{0\}$ such that $I_{\nu}(u)=0$. First, we apply Lemma4.2 with $a=\kappa$ :

$$
2 \lambda \kappa\|x u\|_{L^{2}}^{2}+2 \lambda \int_{\mathbb{R}^{d}}|u(x)|^{2} \ln |u(x)|^{2} d x \geqslant 2 \lambda\|u\|_{L^{2}}^{2}\left(\ln \|u\|_{L^{2}}^{2}-\frac{d}{2} \ln \frac{\pi}{\kappa}\right) .
$$

Using the fact that $\omega^{2}=\kappa^{2}+2 \lambda \kappa$, we thus get

$$
0=I_{\nu}(u) \geqslant\|\nabla u\|_{L^{2}}^{2}+\kappa^{2}\|x u\|_{L^{2}}^{2}+2 \lambda\|u\|_{L^{2}}^{2}\left(\ln \|u\|_{L^{2}}^{2}-\frac{d}{2} \ln \frac{\pi}{\kappa}\right)+2 \nu\|u\|_{L^{2}}^{2} .
$$

Moreover, since the ground state of the harmonic oscillator $-\Delta+\kappa^{2}|x|^{2}$ is $e^{-\kappa|x|^{2} / 2}$, the bottom of the spectrum is $\kappa d$, thus

$$
\|\nabla u\|_{L^{2}}^{2}+\kappa^{2}\|x u\|_{L^{2}}^{2} \geqslant \kappa d\|u\|_{L^{2}}^{2},
$$

with equality if and only if there exists $\mu \in \mathbb{C}$ such that $u(x)=\mu e^{-\kappa|x|^{2} / 2}$. Thus, there holds

$$
0 \geqslant 2 \lambda\|u\|_{L^{2}}^{2}\left(\ln \|u\|_{L^{2}}^{2}-\frac{d}{2} \ln \frac{\pi}{\kappa}+\frac{\kappa d}{2 \lambda}+\frac{\nu}{\lambda}\right) .
$$

Therefore, since $u \neq 0$, we get:

which yields

$$
\ln \|u\|_{L^{2}}^{2}-\frac{d}{2} \ln \frac{\pi}{\kappa}+\frac{\kappa d}{2 \lambda}+\frac{\nu}{\lambda} \leqslant 0,
$$

$$
\|u\|_{L^{2}}^{2} \leqslant \pi^{\frac{d}{2}} \kappa^{-\frac{d}{2}} e^{-\frac{\nu}{\lambda}-\frac{\kappa d}{2 \lambda}} .
$$

Since this inequality holds for all $u \in \Sigma \backslash\{0\}$ such that $I_{\nu}(u)=0$, we get

$$
D(\nu) \geqslant-\lambda \pi^{\frac{d}{2}} \kappa^{-\frac{d}{2}} e^{-\frac{\nu}{\lambda}-\frac{\kappa d}{2 \lambda}} .
$$

Moreover, there is equality in this last inequality if and only if there is equality in all the previous inequalities (4.2), (4.4) and (4.5). From the previous discussion, equality in both (4.2) and (4.4) is equivalent to $u(x)=\mu e^{-\kappa|x|^{2} / 2}$ for some $\mu \in \mathbb{C}$. Putting this condition in the last case of equality shows that $|\mu|^{2}=e^{-\frac{\nu+\kappa d / 2}{\lambda}}$. Therefore, all the inequalities are equalities if and only if there exists $\theta \in\left[0,2 \pi\left[\right.\right.$ such that $u=e^{i \theta} \phi_{\nu}$. The conclusion then readily follows.

REMARK 4.5. If we had to consider equality in 4.2 only, then $\theta$ would be a map from $\mathbb{R}^{d}$ to $\mathbb{R}$, not necessarily constant. On the other hand, equality in (4.4) forces $\theta$ to be constant. 
4.3. Minimizing sequences. As a final preparation for the proof of Theorem 1.5 we characterize minimizing sequences.

LEMMA 4.6. $\left\{u \in \Sigma \backslash\{0\}, I_{\nu}(u)=0\right\}$ is a bounded subset of $\Sigma$.

Proof. Let $u \in \Sigma \backslash\{0\}$ such that $I_{\nu}(u)=0$. By definition of $D(\nu)$, we know that

$$
\|u\|_{L^{2}}^{2} \leqslant-\lambda^{-1} D(\nu) \text {. }
$$

Moreover, using [4.3], we get

$$
\|\nabla u\|_{L^{2}}^{2}+\kappa^{2}\|x u\|_{L^{2}}^{2} \leqslant-2 \lambda\|u\|_{L^{2}}^{2} \ln \|u\|_{L^{2}}^{2}+2 \lambda\|u\|_{L^{2}}^{2}\left(\frac{d}{2} \ln \frac{\pi}{\kappa}+2 \nu\right) .
$$

Using the fact that $-x \ln x \leqslant e^{-1}$ for all $x \geqslant 0$, we get

$$
\|\nabla u\|_{L^{2}}^{2}+\kappa^{2}\|x u\|_{L^{2}}^{2} \leqslant 2 \lambda e^{-1}-2 D(\nu)\left(\frac{d}{2} \ln \frac{\pi}{\kappa}+2 \nu\right),
$$

hence the conclusion, since the $L^{2}$-norm is controlled by $-D(\nu)$.

LeMma 4.7. Let $\left(u_{n}\right)$ be a minimizing sequence for $D(\nu)$. Then there exists $\phi \in \mathcal{G}_{\nu}$ such that, up to a subsequence,

$$
\left\|u_{n}-\phi\right\|_{\Sigma} \underset{n \rightarrow \infty}{\longrightarrow} 0 .
$$

Proof. By definition of $D(\nu)$, we know that

$$
\left\|u_{n}\right\|_{L^{2}}^{2} \underset{n \rightarrow \infty}{\longrightarrow}-\lambda^{-1} D(\nu)
$$

Moreover, Lemmas 4.6 and 4.1 show that there exists $\phi \in \Sigma$ such that, up to a subsequence,

$$
\begin{gathered}
u_{n} \rightarrow \phi \quad \text { weakly in } \Sigma, \\
u_{n} \rightarrow \phi \quad \text { strongly in } L^{2}\left(\mathbb{R}^{d}\right), \\
\lim _{n \rightarrow \infty} \int_{\mathbb{R}^{d}}\left|u_{n}\right|^{2} \ln \left|u_{n}\right|^{2} d x=\int_{\mathbb{R}^{d}}|\phi|^{2} \ln |\phi|^{2} d x .
\end{gathered}
$$

In particular, this yields

$$
\|\phi\|_{L^{2}}=-\lambda^{-1} D(\nu) .
$$

Moreover, using again (4.3), there holds

$$
\left\|\nabla u_{n}\right\|_{L^{2}}^{2}+\kappa^{2}\left\|x u_{n}\right\|_{L^{2}}^{2} \leqslant-2 \lambda\left\|u_{n}\right\|_{L^{2}}^{2} \ln \left\|u_{n}\right\|_{L^{2}}^{2}+2 \lambda\left\|u_{n}\right\|_{L^{2}}^{2}\left(\frac{d}{2} \ln \frac{\pi}{\kappa}-\frac{\nu}{\lambda}\right),
$$

and

$$
\begin{aligned}
&-2 \lambda\left\|u_{n}\right\|_{L^{2}}^{2} \ln \left\|u_{n}\right\|_{L^{2}}^{2}+2 \lambda\left\|u_{n}\right\|_{L^{2}}^{2}\left(\frac{d}{2} \ln \frac{\pi}{\kappa}-\frac{\nu}{\lambda}\right) \\
& \underset{n \rightarrow \infty}{\longrightarrow} 2 D(\nu)\left(\ln \left(-\lambda^{-1} D(\nu)\right)-\frac{d}{2} \ln \frac{\pi}{\kappa}+\frac{\nu}{\lambda}\right)=-\frac{\kappa d}{\lambda} D(\nu),
\end{aligned}
$$

by using Lemma 4.4. Thus, using again (4.4), we get

$$
\begin{aligned}
-\frac{\kappa d}{\lambda} D(\nu)=\kappa d\|\phi\|_{L^{2}} & \leqslant\|\nabla \phi\|_{L^{2}}^{2}+\kappa^{2}\|x \phi\|_{L^{2}}^{2} \\
& \leqslant \liminf \left(\left\|\nabla u_{n}\right\|_{L^{2}}^{2}+\kappa^{2}\left\|x u_{n}\right\|_{L^{2}}^{2}\right) \\
& \leqslant \limsup \left(\left\|\nabla u_{n}\right\|_{L^{2}}^{2}+\kappa^{2}\left\|x u_{n}\right\|_{L^{2}}^{2}\right) \leqslant-\frac{\kappa d}{\lambda} D(\nu) .
\end{aligned}
$$


Therefore, there is equality in all of the above inequalities. In particular,

$$
\|\nabla \phi\|_{L^{2}}^{2}+\kappa^{2}\|x \phi\|_{L^{2}}^{2}=\lim \left(\left\|\nabla u_{n}\right\|_{L^{2}}^{2}+\kappa^{2}\left\|x u_{n}\right\|_{L^{2}}^{2}\right),
$$

which proves that

$$
u_{n} \rightarrow \phi \quad \text { strongly in } \Sigma \text {. }
$$

With all the previous convergences, the convergence of $I_{\nu}\left(u_{n}\right)$ to $I_{\nu}(\phi)$ is now obvious, and therefore $I_{\nu}(\phi)=0$. Since $D(\nu)=-\lambda\|\phi\|_{L^{2}}$, we obtain $\phi \in \mathcal{G}_{\nu}$.

4.4. Orbital stability. Theorem 1.5 is then classically proved by contradiction. Assume that there exist $\varepsilon>0, u_{0 n} \in \Sigma$ and $t_{n} \in \mathbb{R}$ such that

$$
\begin{gathered}
\inf _{\theta \in \mathbb{R}}\left\|u_{0 n}-e^{i \theta} \phi_{\nu}\right\|_{\Sigma} \underset{n \rightarrow \infty}{\longrightarrow} 0, \\
\inf _{\theta \in \mathbb{R}}\left\|u_{n}\left(t_{n}\right)-e^{i \theta} \phi_{\nu}\right\|_{\Sigma}>\varepsilon \quad \text { for any } n \in \mathbb{N} .
\end{gathered}
$$

Set $v_{n}=u_{n}\left(t_{n}\right)$. By (4.6) and the conservation laws, we obtain

$$
\begin{gathered}
\left\|v_{n}\right\|_{L^{2}}^{2}=\left\|u_{0 n}\right\|_{L^{2}}^{2} \underset{n \rightarrow \infty}{\longrightarrow}\left\|\phi_{\nu}\right\|_{L^{2}}^{2}, \\
E\left(v_{n}\right)=E\left(u_{0 n}\right) \underset{n \rightarrow \infty}{\longrightarrow} E\left(\phi_{\nu}\right) .
\end{gathered}
$$

In particular, there also holds

$$
S_{\nu}\left(v_{n}\right) \underset{n \rightarrow \infty}{\longrightarrow} S_{\nu}\left(\phi_{\nu}\right)=D(\nu)
$$

Since $I_{\nu}(f)=2 E(f)+2(\lambda+\nu)\|u\|_{L^{2}}^{2}$,

$$
I_{\nu}\left(v_{n}\right) \underset{n \rightarrow \infty}{\longrightarrow} I_{\nu}\left(\phi_{\nu}\right)=0 .
$$

Next, define the sequence $f_{n}=\rho_{n} v_{n}$ with

$$
\rho_{n}=\exp \left(-\frac{I_{\nu}\left(v_{n}\right)}{2\left\|v_{n}\right\|_{L^{2}}^{2}}\right) .
$$

It is clear that $I_{\nu}\left(f_{n}\right)=0$ and $\lim _{n} \rho_{n}=1$, so that $\left\|v_{n}-f_{n}\right\|_{\Sigma} \rightarrow 0$ and thus $S_{\nu}\left(f_{n}\right) \rightarrow$ $D(\nu)$. Therefore, $\left(f_{n}\right)$ is a minimizing sequence for $D(\nu)$. Applying Lemma 4.7 there exists $\phi \in \mathcal{G}_{\nu}$ such that, up to a subsequence, $f_{n} \rightarrow \phi$ strongly in $\Sigma$. Therefore

$$
v_{n} \rightarrow \phi \quad \text { strongly in } \Sigma \text {. }
$$

Moreover, since $\phi \in \mathcal{G}_{\nu}$, Lemma4.4 gives the existence of $\theta \in\left[0,2 \pi\left[\right.\right.$ such that $\phi=e^{i \theta} \phi_{\nu}$. Therefore, 4.8 contradicts 4.7].

\section{Partial harmonic confinement}

5.1. Preparation of the proof. In this section, we explain how to prove Theorem 1.7 by adapting elements of proof introduced in [16, 22]. Like in Theorem[1.1, we rescale the initial unknown $u$ to a new unknown $v$ through the formula

$$
u\left(t, x^{\prime}, x^{\prime \prime}\right)=\frac{1}{\tau(t)^{d_{2} / 2}} v\left(t, x^{\prime} \frac{x^{\prime \prime}}{\tau(t)}\right) \frac{\left\|u_{0}\right\|_{L^{2}\left(\mathbb{R}^{d}\right)}}{\|\gamma\|_{L^{2}\left(\mathbb{R}^{d_{2}}\right)}} \exp \left(i \frac{\dot{\tau}(t)}{\tau(t)} \frac{\left|x^{\prime \prime}\right|^{2}}{2}\right),
$$

where we emphasize that at this stage, $\gamma$ is a function of the $d_{2}$-dimensional variable $x^{\prime \prime}$. The function $\rho$ involved in Theorem 1.7 is then simply

$$
\rho(t, y)=\int_{\mathbb{R}^{d_{1}}}\left|v\left(t, x^{\prime}, y\right)\right|^{2} d x^{\prime}, \quad y \in \mathbb{R}^{d_{2}} .
$$


We readily check that $(1.1)$ is then equivalent to

$i \partial_{t} v+\frac{1}{2} \Delta_{x^{\prime}} v+\frac{1}{2 \tau(t)^{2}} \Delta_{y} v=\lambda \ln \left(|v|^{2}\right) v+\left(\frac{\omega^{2}}{2}\left|x^{\prime}\right|^{2}+\lambda|y|^{2}\right) v+\theta(t) v, \quad v_{\mid t=0}=\alpha u_{0}$, with $\alpha=\|\gamma\|_{L^{2}\left(\mathbb{R}^{\left.d_{2}\right)}\right.} /\left\|u_{0}\right\|_{L^{2}\left(\mathbb{R}^{d}\right)}$, and where $\theta$ is real-valued and depends on time only,

$$
\theta(t)=-\lambda\left(d_{2} \ln \tau(t)-2 \ln \frac{\left\|u_{0}\right\|_{L^{2}\left(\mathbb{R}^{d}\right)}}{\|\gamma\|_{L^{2}\left(\mathbb{R}^{d_{2}}\right)}}\right)=-\lambda\left(d_{2} \ln \tau(t)-2 \ln \frac{\left\|u_{0}\right\|_{L^{2}\left(\mathbb{R}^{d}\right)}}{\pi^{d_{2} / 2}}\right) .
$$

Changing $v\left(t, x^{\prime}, y\right)$ to $v\left(t, x^{\prime}, y\right) e^{i \int_{0}^{t} \theta(s) d s}$ does not affect the function $\rho$ involved in the statement of Theorem 1.7 and removes the last term from the above equation, so we may assume that $v$ solves

(5.2) $i \partial_{t} v+\frac{1}{2} \Delta_{x^{\prime}} v+\frac{1}{2 \tau(t)^{2}} \Delta_{y} v=\lambda \ln \left(|v|^{2}\right) v+\left(\frac{\omega^{2}}{2}\left|x^{\prime}\right|^{2}+\lambda|y|^{2}\right) v, \quad v_{\mid t=0}=v_{0}$,

where $v_{0}$ is such that $\left\|v_{0}\right\|_{L^{2}\left(\mathbb{R}^{d}\right)}=\|\gamma\|_{L^{2}\left(\mathbb{R}^{\left.d_{2}\right)}\right.}$. The equation (5.2) is still Hamiltonian, but since it is no longer autonomous, we have a dissipated energy: setting

$$
\begin{aligned}
\mathcal{E}(t)= & \frac{1}{2}\left\|\nabla_{x^{\prime}} v\right\|_{L^{2}\left(\mathbb{R}^{d}\right)}^{2}+\frac{1}{2 \tau(t)^{2}}\left\|\nabla_{y} v\right\|_{L^{2}\left(\mathbb{R}^{d}\right)}^{2}+\lambda \int_{\mathbb{R}^{d}}\left|v\left(t, x^{\prime}, y\right)\right|^{2} \ln \left|v\left(t, x^{\prime}, y\right)\right|^{2} d x^{\prime} d y \\
& +\int_{\mathbb{R}^{d}}\left(\frac{\omega^{2}}{2}\left|x^{\prime}\right|^{2}+\lambda|y|^{2}\right)\left|v\left(t, x^{\prime}, y\right)\right|^{2} d x^{\prime} d y,
\end{aligned}
$$

we have

This implies crucial a priori estimates:

$$
\frac{d \mathcal{E}}{d t}=-\frac{\dot{\tau}}{\tau^{3}}\left\|\nabla_{y} v\right\|_{L^{2}\left(\mathbb{R}^{d}\right)}^{2} \leqslant 0 .
$$

Lemma 5.1. Let $u_{0} \in \Sigma, \lambda, \omega>0$. There exists $C>0$ such that

$$
\begin{aligned}
& \sup _{t \geqslant 0} \int_{\mathbb{R}^{d}}\left(1+\left|x^{\prime}\right|^{2}+|y|^{2}+\left.|\ln | v\left(t, x^{\prime}, y\right)\right|^{2} \mid\right)\left|v\left(t, x^{\prime}, y\right)\right|^{2} d x^{\prime} d y \leqslant C, \\
& \sup _{t \geqslant 0}\left(\frac{1}{2}\left\|\nabla_{x^{\prime}} v\right\|_{L^{2}\left(\mathbb{R}^{d}\right)}^{2}+\frac{1}{2 \tau(t)^{2}}\left\|\nabla_{y} v\right\|_{L^{2}\left(\mathbb{R}^{d}\right)}^{2}\right) \leqslant C .
\end{aligned}
$$

In addition,

$$
\int_{0}^{\infty} \frac{\dot{\tau}(t)}{\tau(t)^{3}}\left\|\nabla_{y} v(t)\right\|_{L^{2}\left(\mathbb{R}^{d}\right)}^{2}<\infty
$$

SKETCH OF THE PROOF. We resume the main lines of the proof of [16, Lemma 4.1]. Write $\mathcal{E}=\mathcal{E}_{+}-\mathcal{E}_{-}$, where

$$
\begin{aligned}
\mathcal{E}_{+}= & \frac{1}{2}\left\|\nabla_{x^{\prime}} v\right\|_{L^{2}}^{2}+\frac{1}{2 \tau(t)^{2}}\left\|\nabla_{y} v\right\|_{L^{2}}^{2}+\int_{\mathbb{R}^{d}}\left(\frac{\omega^{2}}{2}\left|x^{\prime}\right|^{2}+\lambda|y|^{2}\right)\left|v\left(t, x^{\prime}, y\right)\right|^{2} d x^{\prime} d y \\
& +\lambda \int_{\left|v\left(t, x^{\prime}, y\right)\right|^{2} \geqslant 1}\left|v\left(t, x^{\prime}, y\right)\right|^{2} \ln \left|v\left(t, x^{\prime}, y\right)\right|^{2} d x^{\prime} d y .
\end{aligned}
$$

Note that $\mathcal{E}_{+}$is the sum of non-negative terms only. Since $\mathcal{E}$ is non-increasing, for $t \geqslant 0$,

$$
\begin{aligned}
\mathcal{E}_{+}(t) & \leqslant \mathcal{E}(0)+\mathcal{E}_{-}(t)=\mathcal{E}(0)+\lambda \int_{|v| \leqslant 1}\left|v\left(t, x^{\prime}, y\right)\right|^{2} \ln \frac{1}{\left|v\left(t, x^{\prime}, y\right)\right|^{2}} d x^{\prime} d y \\
& \lesssim 1+\int_{\mathbb{R}^{d}}\left|v\left(t, x^{\prime}, y\right)\right|^{2-\varepsilon} d x^{\prime} d y
\end{aligned}
$$


for any $\varepsilon>0$ sufficiently small. Then $\|v\|_{L^{2-\varepsilon}} \lesssim\|v\|_{L^{2}}^{1-\delta(\varepsilon)}\left\|\left(\left|x^{\prime}\right|+|y|\right) v\right\|_{L^{2}}^{\delta(\varepsilon)}$ with $\delta(\varepsilon) \rightarrow 0$ as $\varepsilon \rightarrow 0$. In view of the conservation of the $L^{2}$-norm of $v$ (which is equal to the $L^{2}$-norm of $u$ ), we infer, for $0<\varepsilon \ll 1, \mathcal{E}_{-} \lesssim \mathcal{E}_{+}^{1 / 2}$ (for instance), hence

$$
\mathcal{E}_{+}(t) \lesssim 1, \quad \forall t \geqslant 0 \text {. }
$$

Therefore, each term in $\mathcal{E}_{+}$is bounded, and so is $\mathcal{E}_{-}$.

Since $\mathcal{E}$ is bounded, the integral of $\dot{\mathcal{E}}$ is bounded, hence the last part of the lemma.

5.2. Convergence of momenta. The proof of (1.7) stems from the same arguments as in [16].

5.2.1. Center of mass. Introduce

$$
I_{1}(t):=\operatorname{Im} \int_{\mathbb{R}^{d}} \bar{v}\left(t, x^{\prime}, y\right) \nabla_{y} v\left(t, x^{\prime}, y\right) d x^{\prime} d y, \quad I_{2}(t):=\int_{\mathbb{R}^{d}} y\left|v\left(t, x^{\prime}, y\right)\right|^{2} d x^{\prime} d y .
$$

We compute

$$
\dot{I}_{1}=-2 \lambda I_{2}, \quad \dot{I}_{2}=\frac{1}{\tau^{2}(t)} I_{1}
$$

and so $\tilde{I}_{2}:=\tau I_{2}$ satisfies $\ddot{\tilde{I}}_{2}=0$. Since $\tau(t) \sim 2 t \sqrt{\lambda \ln t}$ as $t \rightarrow \infty$, we infer that $I_{2}(t)=\mathcal{O}(\ln t)^{-1 / 2}$.

5.2.2. Second order momentum. Rewriting in terms of $v$ the conservation of the energy for $u$, 1.2), we find:

$$
\begin{aligned}
E\left(u_{0}\right)= & \frac{1}{2}\left\|\nabla_{x^{\prime}} v\right\|_{L^{2}\left(\mathbb{R}^{d}\right)}^{2}+\frac{1}{2 \tau(t)^{2}}\left\|\nabla_{y} v\right\|_{L^{2}\left(\mathbb{R}^{d}\right)}^{2}+\frac{(\dot{\tau})^{2}}{2} \int_{\mathbb{R}^{d}}|y|^{2}\left|v\left(t, x^{\prime}, y\right)\right|^{2} d x^{\prime} d y \\
& +\frac{\dot{\tau}}{\tau} \operatorname{Im} \int_{\mathbb{R}^{d}} \bar{v}\left(t, x^{\prime}, y\right) y \cdot \nabla_{y} v\left(t, x^{\prime}, y\right) d x^{\prime} d y+\frac{\omega^{2}}{2} \int_{\mathbb{R}^{d}}\left|x^{\prime}\right|^{2}\left|v\left(t, x^{\prime}, y\right)\right|^{2} d x^{\prime} d y \\
& +\lambda \int_{\mathbb{R}^{d}}\left|v\left(t, x^{\prime}, y\right)\right|^{2} \ln \left|v\left(t, x^{\prime}, y\right)\right|^{2} d x^{\prime} d y-\lambda d_{2} \pi^{d_{2}} \ln \tau \\
& +2 \lambda\left\|u_{0}\right\|_{L^{2}\left(\mathbb{R}^{d}\right)}^{2} \ln \left(\frac{\left\|u_{0}\right\|_{L^{2}\left(\mathbb{R}^{d}\right)}}{\pi^{d_{2} / 2}}\right) .
\end{aligned}
$$

In view of Lemma 5.1, the first, second, fifth, sixth, and last terms on the right-hand side are bounded in time. In view of Lemma 5.1 and Cauchy-Schwarz inequality, the fourth term is $\mathcal{O}(\dot{\tau})=\mathcal{O}(\sqrt{\ln t})$, and we obtain

$$
\frac{(\dot{\tau})^{2}}{2} \int_{\mathbb{R}^{d}}|y|^{2}\left|v\left(t, x^{\prime}, y\right)\right|^{2} d x^{\prime} d y-\lambda d_{2} \pi^{d_{2}} \ln \tau=\mathcal{O}(\sqrt{\ln t}),
$$

which yields, since $(\dot{\tau})^{2}=4 \lambda \ln \tau$ (multiply 11.3 by $\dot{\tau}$ and integrate),

$$
\int_{\mathbb{R}^{d}}|y|^{2}\left|v\left(t, x^{\prime}, y\right)\right|^{2} d x^{\prime} d y=\frac{d_{2}}{2} \pi^{d_{2}}+\mathcal{O}\left(\frac{1}{\sqrt{\ln t}}\right)=\int_{\mathbb{R}^{d_{2}}}|y|^{2} \gamma(y)^{2} d y+\mathcal{O}\left(\frac{1}{\sqrt{\ln t}}\right),
$$

hence (1.7).

5.3. Convergence of the profile. To prove the end of Theorem 1.7 we use a Madelung transform: define $R, J_{1}$ and $J_{2}$ by

$$
\begin{aligned}
& R\left(t, x^{\prime}, y\right)=\left|v\left(t, x^{\prime}, y\right)\right|^{2}, \quad J_{1}\left(t, x^{\prime}, y\right)=\operatorname{Im}\left(\bar{v}\left(t, x^{\prime}, y\right) \nabla_{x^{\prime}} v\left(t, x^{\prime}, y\right)\right), \\
& J_{2}\left(t, x^{\prime}, y\right)=\operatorname{Im}\left(\bar{v}\left(t, x^{\prime}, y\right) \nabla_{y} v\left(t, x^{\prime}, y\right)\right) .
\end{aligned}
$$


We check that (5.2) implies

$$
\begin{aligned}
\partial_{t} R+\nabla_{x^{\prime}} \cdot J_{1}+\frac{1}{\tau(t)^{2}} \nabla_{y} \cdot J_{2}=0 & \\
\partial_{t} J_{2}+\lambda \nabla_{y} R+2 \lambda y R= & \frac{1}{4 \tau(t)^{2}} \Delta_{y} \nabla_{y} R-\frac{1}{\tau(t)^{2}} \nabla_{y} \cdot\left(\operatorname{Re}\left(\nabla_{y} v \otimes \nabla_{y} \bar{v}\right)\right) \\
& +\frac{1}{2} \nabla_{x^{\prime}} \cdot \operatorname{Re}\left(\bar{v}\left(\left(\nabla_{x^{\prime}} \otimes \nabla_{y}\right) v\right)-\nabla_{x^{\prime}} \bar{v} \otimes \nabla_{y} v\right) .
\end{aligned}
$$

We do not write the evolution law for $J_{1}$, as it is not needed for the argument.

REMARK 5.2. Despite the fact that $v(t)$ might not be $H^{2}$, the term $\bar{v}\left(\nabla_{x^{\prime}} \otimes \nabla_{y}\right) v$ is still well defined in $\mathcal{D}^{\prime}\left((0, \infty) \times \mathbb{R}^{d}\right)$ owing to the fact that $v$ is in $L_{\mathrm{loc}}^{\infty}\left((0, \infty) ; H^{1}\right)$ and to the relation:

$$
\bar{v}\left(\nabla_{x^{\prime}} \otimes \nabla_{y}\right) v=\nabla_{y}\left(\bar{v} \nabla_{x^{\prime}} v\right)-\nabla_{y} \bar{v} \otimes \nabla_{x^{\prime}} v
$$

We note that

$$
\rho(t, y)=\int_{\mathbb{R}^{d_{1}}} R\left(t, x^{\prime}, y\right) d x^{\prime}
$$

and we set

$$
j(t, y)=\int_{\mathbb{R}^{d_{1}}} J_{2}\left(t, x^{\prime}, y\right) d x^{\prime} .
$$

These new unknowns solve, in $\mathcal{D}^{\prime}\left((0, \infty) \times \mathbb{R}^{d_{2}}\right)$ :

$$
\left\{\begin{array}{l}
\partial_{t} \rho+\frac{1}{\tau(t)^{2}} \nabla_{y} \cdot j=0 \\
\partial_{t} j+\lambda \nabla_{y} \rho+2 \lambda y \rho=\frac{1}{4 \tau(t)^{2}} \Delta_{y} \nabla_{y} \rho-\frac{1}{\tau(t)^{2}} \nabla_{y} \cdot \mu
\end{array}\right.
$$

where

$$
\mu(t, y)=\int_{\mathbb{R}^{d_{1}}} \operatorname{Re}\left(\nabla_{y} v \otimes \nabla_{y} \bar{v}\right)\left(t, x^{\prime}, y\right) d x^{\prime} .
$$

At this stage, in view of the a priori estimates provided by Lemma 5.1, we have the same ingredients are those needed in [16. Section 5.3] and [22. Lemma 4.4], hence the weak convergence and the estimate of the Wasserstein distance in Theorem 1.7.

\section{Repulsive harmonic potential}

In this section, we consider (1.5), that is

$$
i \partial_{t} u+\frac{1}{2} \Delta u=-\omega^{2} \frac{|x|^{2}}{2} u+\lambda u \ln \left(|u|^{2}\right), \quad u_{\mid t=0}=u_{0},
$$

for $x \in \mathbb{R}^{d}$ and $\omega, \lambda>0$.

6.1. Analysis of the dispersion. We first resume the analysis started in Section 3.3 in the Gaussian case, and consider $\tau$ solving (3.5). We discuss the dependence upon initial data at the end. 
6.1.1. Direct error estimate. Let $\tau_{\text {eff }}^{T}$ solve

$$
\ddot{\tau}_{\text {eff }}^{T}=\omega^{2} \tau_{\text {eff }}^{T}, \quad \tau_{\text {eff }}^{T}(T)=\tau(T), \dot{\tau}_{\text {eff }}^{T}(T)=\dot{\tau}(T) .
$$

Consider the error $e_{T}=\tau-\tau_{\text {eff }}^{T}$. It solves

$$
\ddot{e}_{T}-\omega^{2} e_{T}=\frac{2 \lambda}{\tau}+\frac{1}{\tau^{3}},
$$

and Duhamel's formula reads

$$
e_{T}(t)=2 \lambda \int_{T}^{t} \frac{\sinh (\omega(t-s))}{\omega} \frac{d s}{\tau(s)}+\int_{T}^{t} \frac{\sinh (\omega(t-s))}{\omega} \frac{d s}{\tau(s)^{3}} .
$$

In view of 3.7), for $t \geqslant T \gg 1$,

$$
\left|e_{T}(t)\right| \leqslant \varepsilon \int_{T}^{t} \frac{\sinh (\omega(t-s))}{\omega} d s \leqslant \frac{\varepsilon}{\omega^{2}} \cosh (\omega(t-T)) .
$$

On the other hand,

$$
\tau_{\text {eff }}^{T}(t) \underset{t \rightarrow \infty}{\sim} \frac{1}{2}\left(\tau(T)+\frac{\dot{\tau}(T)}{\omega}\right) e^{\omega(t-T)} .
$$

We infer from 3.7) that $\tau$ grows exponentially in time, since $\tau(T)+\frac{\dot{\tau}(T)}{\omega} \gg \varepsilon$. In view of (3.6), $\dot{\tau}$ also grows exponentially in time:

$$
e^{\omega t} \gtrsim \tau(t) \gtrsim e^{\omega t}, \quad e^{\omega t} \gtrsim \dot{\tau}(t) \gtrsim e^{\omega t}, \quad t>0 .
$$

6.1.2. Analysis of the main $O D E$. Setting $\mu(t)=\tau(t) e^{-\omega t}$, we prove that $\mu(t) \rightarrow \mu_{\infty}$ as $t \rightarrow \infty$. It solves

$$
\ddot{\mu}+2 \omega \dot{\mu}=\frac{2 \lambda}{\mu} e^{-2 \omega t}+\frac{1}{\mu^{3}} e^{-4 \omega t},
$$

hence

$$
\frac{d}{d t}\left(\dot{\mu} e^{2 \omega t}\right)=\frac{2 \lambda}{\mu}+\frac{1}{\mu^{3}} e^{-2 \omega t}>0 .
$$

We know from the previous section that

$$
1 \lesssim \mu(t) \lesssim 1
$$

and the previous bound becomes

$$
\frac{d}{d t}\left(\dot{\mu} e^{2 \omega t}\right) \gtrsim 1
$$

The map $t \mapsto \dot{\mu}(t) e^{2 \omega t}$ is increasing, and

$$
\dot{\mu}(t) e^{2 \omega t} \gtrsim t-c \underset{t \rightarrow \infty}{\longrightarrow} \infty .
$$

Then (6.2) implies

$$
\ddot{\mu}(t) e^{2 \omega t} \lesssim-t+c \underset{t \rightarrow \infty}{\longrightarrow}-\infty .
$$

Therefore, for $t$ sufficiently large, $\dot{\mu}$ is positive decreasing, hence has a non-negative limit as $t \rightarrow \infty$, and $\ddot{\mu} \in L^{1}$. But 6.2 implies that $\dot{\mu}$ and $\ddot{\mu}$ are simultaneously $L^{1}$, so $\dot{\mu} \in L^{1}$ and the limit of $\dot{\mu}$ has to be zero. This yields the existence of $\mu_{\infty}>0$ such that

$$
\mu(t) \underset{t \rightarrow \infty}{\longrightarrow} \mu_{\infty}, \text { hence } \tau(t) \underset{t \rightarrow \infty}{\sim} \mu_{\infty} e^{\omega t} .
$$

In view of [3.6, and since $\dot{\tau}>0$ (at least for $t \gg 1$ ), we infer

$$
\dot{\tau}(t) \underset{t \rightarrow \infty}{\sim} \omega \mu_{\infty} e^{\omega t} .
$$


6.1.3. Dependence of $\mu_{\infty}$. First, we get another integral expression for $\tau$. Define $F(t):=\frac{2 \lambda}{\tau(t)}+\frac{1}{\tau(t)^{3}}$. Then there holds

$$
\ddot{\tau}-\omega^{2} \tau=F
$$

which leads to

$$
\frac{d}{d t}\left(e^{\omega t}(\dot{\tau}-\omega \tau)\right)=F(t) e^{\omega t}
$$

and thus

$$
e^{\omega t}(\dot{\tau}-\omega \tau)=(\dot{\tau}(0)-\omega \tau(0))+\int_{0}^{t} F(s) e^{\omega s} d s
$$

Therefore, we get

$$
\tau(t)=e^{\omega t}\left[\tau(0)+\frac{1-e^{-2 \omega t}}{2 \omega}(\dot{\tau}(0)-\omega \tau(0))+\int_{0}^{t} e^{-2 \omega s} \int_{0}^{s} F(r) e^{\omega r} d r d s\right]
$$

After some easier computations, we get

(6.4) $\tau(t)=\tau(0) \cosh (\omega t)+\frac{\dot{\tau}(0)}{\omega} \sinh (\omega t)+\frac{e^{\omega t}}{2 \omega} \int_{0}^{t} e^{-\omega r} F(r) d r$

$$
-\frac{e^{-\omega t}}{2 \omega} \int_{0}^{t} F(r) e^{\omega r} d r
$$

We also already know that $\tau(t) \sim \mu_{\infty} e^{\omega t}$, so in particular $F(t) \sim \frac{2 \lambda}{\mu_{\infty}} e^{-\omega t}$, and thus

$$
\int_{0}^{t} F(r) e^{\omega r} d r=\mathcal{O}(t), \quad \int_{0}^{\infty} F(r) e^{-\omega r} d r<\infty .
$$

Therefore, 6.4 leads to an expression for $\mu_{\infty}$ :

$$
\mu_{\infty}=\tau(0)+\frac{\dot{\tau}(0)}{\omega}+\frac{1}{2 \omega} \int_{0}^{\infty} e^{-\omega r}\left(\frac{2 \lambda}{\tau(r)}+\frac{1}{\tau(r)^{3}}\right) d r
$$

Moreover, from the fact that $F \geqslant 0$ and $e^{\omega t} e^{-\omega r}-e^{-\omega t} e^{\omega r} \geqslant 0$ for all $0 \leqslant r \leqslant t, 6.4$ also gives for all $t \geqslant 0$ :

$$
\tau(t) \geqslant \tau(0) \cosh (\omega t)+\frac{\dot{\tau}(0)}{\omega} \sinh (\omega t) .
$$

Assume $\dot{\tau}(0)=\tau_{1} \geqslant 0$ and $\tau(0)=\tau_{0}>0$. Then, the last term in the right-hand side of 6.5) can be estimated. First:

$$
\begin{aligned}
0 \leqslant \int_{0}^{\infty} e^{-\omega r} \frac{2 \lambda}{\tau(r)} d r & \leqslant \int_{0}^{\infty} e^{-\omega r} \frac{2 \lambda}{\tau_{0} \cosh (\omega r)+\frac{\tau_{1}}{\omega} \sinh (\omega r)} d r \\
& \leqslant \int_{0}^{\infty} e^{-2 \omega r} \frac{4 \lambda}{\tau_{0}\left(1+e^{-2 \omega r}\right)+\frac{\tau_{1}}{\omega}\left(1-e^{-2 \omega r}\right)} d r \\
& \leqslant-\frac{2 \lambda}{\omega\left(\tau_{0}-\frac{\tau_{1}}{\omega}\right)}\left[\ln \left(\tau_{0}\left(1+e^{-2 \omega r}\right)+\frac{\tau_{1}}{\omega}\left(1-e^{-2 \omega r}\right)\right)\right]_{0}^{\infty} \\
& \leqslant \frac{2 \lambda}{\omega\left(\frac{\tau_{1}}{\omega}-\tau_{0}\right)} \ln \left(1+\frac{\frac{\tau_{1}}{\omega}-\tau_{0}}{2 \tau_{0}}\right)
\end{aligned}
$$


Then, we also have

$$
\begin{aligned}
0 \leqslant \int_{0}^{\infty} e^{-\omega r} \frac{1}{(\tau(r))^{3}} d r & \leqslant \int_{0}^{\infty} e^{-\omega r} \frac{1}{\left(\tau_{0} \cosh (\omega r)+\frac{\tau_{1}}{\omega} \sinh (\omega r)\right)^{3}} d r \\
& \leqslant \int_{0}^{\infty} e^{2 \omega r} \frac{16 \lambda}{\left(\tau_{0}\left(1+e^{2 \omega r}\right)+\frac{\tau_{1}}{\omega}\left(e^{2 \omega r}-1\right)\right)^{3}} d r \\
& \leqslant-\frac{4 \lambda}{\omega\left(\tau_{0}+\frac{\tau_{1}}{\omega}\right)}\left[\left(\tau_{0}\left(1+e^{2 \omega r}\right)+\frac{\tau_{1}}{\omega}\left(e^{2 \omega r}-1\right)\right)^{-2}\right]_{0}^{\infty} \\
& \leqslant \frac{2 \lambda}{\omega\left(\frac{\tau_{1}}{\omega}+\tau_{0}\right) \tau_{0}} .
\end{aligned}
$$

Then, as soon as $\tau_{0}$ is fixed, we get an expansion of $\mu_{\infty}$ with respect to $\tau_{1}$ with 6.5):

$$
\mu_{\infty}=\frac{\tau_{1}}{\omega}+\tau_{0}+\mathcal{O}\left(\frac{\ln \tau_{1}}{\tau_{1}}\right) .
$$

6.2. Back to the PDE. We now address the general case, in the sense that $u_{0}$ need not be Gaussian. As announced in Proposition 1.8, change the unknown $u$ to $v$, through the formula

$$
u(t, x)=\frac{1}{\tau_{-}(t)^{d / 2}} v\left(t, \frac{x}{\tau_{-}(t)}\right) \exp \left(i \frac{\dot{\tau}_{-}(t)}{\tau_{-}(t)} \frac{|x|^{2}}{2}\right),
$$

with $\tau_{-}$solution to (1.6), that is, 3.5 where the last term is discarded (this simplifies a little bit the computations, and the discarded term brings no extra information any way). Then (3.2) is equivalent, up to an irrelevant time dependent phase (like previously), to

$$
i \partial_{t} v+\frac{1}{2 \tau_{-}^{2}} \Delta v=\lambda|y|^{2} v+\lambda v \ln |v|^{2}, \quad v_{\mid t=0}=u_{0}
$$

provided that we assume

$$
\tau_{-}(0)=1, \quad \dot{\tau}_{-}(0)=0 .
$$

6.2.1. Hamiltonian structure and consequences. Set

$$
\mathcal{E}(t)=\underbrace{\frac{1}{2 \tau_{-}(t)^{2}}\|\nabla v(t)\|_{L^{2}}^{2}}_{=: \mathcal{E}_{\text {kin }}(t)}+\lambda \int_{\mathbb{R}^{d}}|y|^{2}|v(t, y)|^{2} d y+\lambda \int_{\mathbb{R}^{d}}|v(t, y)|^{2} \ln |v(t, y)|^{2} d y
$$

We compute

$$
\dot{\mathcal{E}}(t)=-2 \frac{\dot{\tau}_{-}(t)}{\tau_{-}(t)} \mathcal{E}_{\text {kin }}(t) .
$$

We readily infer, with the same proof as in the case $\omega=0$ given in [16. Lemma 4.1], as already sketched in the proof of Lemma 5.1 .

LEMMA 6.1. For $u_{0} \in \Sigma$ and $\lambda>0$, there holds

$$
\sup _{t \geqslant 0}\left(\int_{\mathbb{R}^{d}}\left(1+|y|^{2}+\left.|\ln | v(t, y)\right|^{2} \mid\right)|v(t, y)|^{2} d y+\frac{1}{\tau_{-}(t)^{2}}\|\nabla v(t)\|_{L^{2}\left(\mathbb{R}^{d}\right)}^{2}\right)<\infty
$$

and

$$
\int_{0}^{\infty} \frac{\dot{\tau}_{-}(t)}{\tau_{-}^{3}(t)}\|\nabla v(t)\|_{L^{2}\left(\mathbb{R}^{d}\right)}^{2} d t<\infty
$$


SKETCH OF THE PROOF. Write $\mathcal{E}=\mathcal{E}_{+}-\mathcal{E}_{-}$, where

$$
\mathcal{E}_{+}=\frac{1}{2 \tau_{-}(t)^{2}}\|\nabla v(t)\|_{L^{2}}^{2}+\lambda \int_{\mathbb{R}^{d}}|y|^{2}|v(t, y)|^{2} d y+\lambda \int_{|v|>1}|v(t, y)|^{2} \ln |v(t, y)|^{2} d y .
$$

Note that $\mathcal{E}_{+}$is the sum of non-negative terms only. Since $\mathcal{E}$ is non-increasing, for $t \geqslant 0$,

$$
\begin{aligned}
\mathcal{E}_{+}(t) & \leqslant \mathcal{E}(0)+\mathcal{E}_{-}(t)=\mathcal{E}(0)+\lambda \int_{|v| \leqslant 1}|v(t, y)|^{2} \ln \frac{1}{|v(t, y)|^{2}} d y \\
& \lesssim 1+\int_{\mathbb{R}^{d}}|v(t, y)|^{2-\varepsilon} d y
\end{aligned}
$$

for any $\varepsilon>0$ sufficiently small. Then $\|v\|_{L^{2-\varepsilon}} \lesssim\|v\|_{L^{2}}^{1-\delta(\varepsilon)}\|y v\|_{L^{2}}^{\delta(\varepsilon)}$ with $\delta(\varepsilon) \rightarrow 0$ as $\varepsilon \rightarrow 0$, and we conclude like in the proof of Lemma 5.1 .

Since the $L^{2}$-norm of $v$ is independent of time, the boundedness of the momentum of $v$ is an evidence that $v$ is not dispersive, while the boundedness of $|v|^{2}$ in LlogL is an evidence that $v$ does not grow to infinity. Therefore $\tau_{-}$describes the dispersive rate of any solution to (1.5).

6.2.2. Center of mass. Introduce

$$
I_{1}(t):=\operatorname{Im} \int_{\mathbb{R}^{d}} \bar{v}(t, y) \nabla v(t, y) d y, \quad I_{2}(t):=\int_{\mathbb{R}^{d}} y|v(t, y)|^{2} d y .
$$

We compute:

$$
\dot{I}_{1}=-2 \lambda I_{2}, \quad \dot{I}_{2}=\frac{1}{\tau_{-}^{2}(t)} I_{1}
$$

Set $\tilde{I}_{2}:=\tau_{-} I_{2}$. We compute, in view of (3.5),

$$
\frac{d^{2}}{d t^{2}} \tilde{I}_{2}=\omega^{2} \tilde{I}_{2}, \quad \text { hence } \quad \tau_{-}(t) I_{2}(t)=a_{0} \cosh (\omega t)+b_{0} \frac{\sinh (\omega t)}{\omega} .
$$

In view of 6.97,

$$
a_{0}=I_{2}(0), \quad b_{0}=\dot{I}_{2}(0)=I_{1}(0)
$$

We infer

$$
I_{2}(t) \underset{t \rightarrow \infty}{\sim} \frac{a_{0}+b_{0}}{2} \frac{e^{\omega t}}{\tau_{-}(t)} \underset{t \rightarrow \infty}{\sim} \frac{a_{0}+b_{0}}{2 \mu_{\infty}}=\frac{I_{1}(0)+I_{2}(0)}{2 \mu_{\infty}} .
$$

Unlike in the case $\omega=0$ considered in [16, 22], the asymptotic center of mass of $v$ is not zero in general (while it is always zero in the context of Theorem 1.1); this is like in the scattering case, where the asymptotic profile has no reason to be centered at the origin (as shown by the existence of wave operators).

Integrating in time the first equation in 6.10 , we infer

$$
I_{1}(t) \underset{t \rightarrow \infty}{\sim}-\lambda t \frac{I_{1}(0)+I_{2}(0)}{\mu_{\infty}},
$$

provided that $I_{1}(0)+I_{2}(0) \neq 0$. This suggests that $v$ is oscillatory. 
6.2.3. More on large time behavior. At this stage, we have established two differences with the dynamics of $\log$ NLS (without potential): the dispersion is the one dictated by the repulsive harmonic potential, that is, exponential, and in the dispersive frame (working with $v$ ), the asymptotic center of mass is not necessarily zero.

The next natural question would be to decide between a general asymptotic behavior for $|v|$ (like in the case $\omega=0$ ) or a complete scattering theory. We are not able to fully validate the second option, which is the most likely in view of the result on the center of mass, but the Gaussian case shows that no universal profile must be expected for the large time behavior of $|v|$.

To see this, consider $d=1$ and two Gaussian initial data

$$
u_{01}(x)=e^{-x^{2} / 2+i \beta_{1} x^{2} / 2}, \quad u_{02}(x)=e^{-x^{2} / 2+i \beta_{2} x^{2} / 2}, \quad \beta_{j}>0 .
$$

In other words, we start from two distinct Gaussian data, whose moduli (hence all Lebesgue norms and momenta, for instance) are equal. The corresponding solutions $u_{1}$ and $u_{2}$ are given by the formula presented in Section 3.3 , boiling the description down to the analysis of the ODE (3.5):

$$
u_{j}(t, x)=b_{j}(t) e^{-x^{2} /\left(2 \tau_{j}(t)^{2}\right)+i \dot{\tau}_{j}(t) x^{2} /\left(2 \tau_{j}(t)\right)}, \quad \tau_{j}(t) \underset{t \rightarrow \infty}{\sim} \mu_{\infty, j} e^{\omega t} .
$$

In view of Section 6.1 we have

$$
\mu_{\infty, j}=1+\frac{\beta_{j}}{\omega}+\mathcal{O}\left(\frac{\ln \beta_{j}}{\beta_{j}}\right) \text { as } \beta_{j} \rightarrow \infty,
$$

and so for $\beta_{2} \gg \beta_{1} \gg 1$, the corresponding functions $v_{j}$ have different (asymptotic centers of mass and) asymptotic profiles. The fact that $\left|v_{j}\right|^{2}$ converges strongly in $L^{1}$ to the corresponding limiting Gaussian is straightforward; see e.g. [16. Corollary 1.11].

\section{References}

[1] C. Ané, S. Blachère, D. Chafaï, P. Fougères, I. Gentil, F. Malrieu, C. Roberto, and G. Scheffer. Sur les inégalités de Sobolev logarithmiques, volume 10 of Panoramas et Synthèses [Panoramas and Syntheses]. Société Mathématique de France, Paris, 2000. With a preface by Dominique Bakry and Michel Ledoux.

[2] P. Antonelli, R. Carles, and J. Drumond Silva. Scattering for nonlinear Schrödinger equation under partial harmonic confinement. Comm. Math. Phys., 334(1):367-396, 2015.

[3] A. H. Ardila. Orbital stability of Gausson solutions to logarithmic Schrödinger equations. Electron. J. Differential Equations, pages Paper No. 335, 9, 2016.

[4] A. H. Ardila, L. Cely, and M. Squassina. Logarithmic Bose-Einstein condensates with harmonic potential. Asymptotic Anal., 116(1):27-40, 2020.

[5] A. V. Avdeenkov and K. G. Zloshchastiev. Quantum Bose liquids with logarithmic nonlinearity: Selfsustainability and emergence of spatial extent. J. Phys. B: Atomic, Molecular Optical Phys., 44(19):195303, 2011.

[6] W. Bao, R. Carles, C. Su, and Q. Tang. Error estimates of a regularized finite difference method for the logarithmic Schrödinger equation. SIAM J. Numer. Anal., 57(2):657-680, 2019.

[7] J. Bellazzini, N. Boussaïd, L. Jeanjean, and N. Visciglia. Existence and stability of standing waves for supercritical NLS with a partial confinement. Comm. Math. Phys., 353(1):229-251, 2017.

[8] I. Białynicki-Birula and J. Mycielski. Nonlinear wave mechanics. Ann. Physics, 100(1-2):62-93, 1976.

[9] I. Białynicki-Birula and J. Mycielski. Gaussons: Solitons of the logarithmic Schrödinger equation. Special issue on solitons in physics, Phys. Scripta, 20:539-544, 1979.

[10] B. Bouharia. Stability of logarithmic Bose-Einstein condensate in harmonic trap. Modern Physcis Letters B, 29(01):1450260, 2015.

[11] H. Brézis and E. Lieb. A relation between pointwise convergence of functions and convergence of functionals. Proc. Amer. Math. Soc., 88(3):486-490, 1983.

[12] H. Buljan, A. Šiber, M. Soljačić, T. Schwartz, M. Segev, and D. Christodoulides. Incoherent white light solitons in logarithmically saturable noninstantaneous nonlinear media. Phys. Rev. E, 68(3):036607, 2003. 
[13] R. Carles. Nonlinear Schrödinger equations with repulsive harmonic potential and applications. SIAM J. Math. Anal., 35(4):823-843, 2003.

[14] R. Carles. Global existence results for nonlinear Schrödinger equations with quadratic potentials. Discrete Contin. Dyn. Syst., 13(2):385-398, 2005.

[15] R. Carles. Nonlinear Schrödinger equation with time dependent potential. Commun. Math. Sci., 9(4):937964, 2011.

[16] R. Carles and I. Gallagher. Universal dynamics for the defocusing logarithmic Schrödinger equation. Duke Math. J., 167(9):1761-1801, 2018.

[17] T. Cazenave. Stable solutions of the logarithmic Schrödinger equation. Nonlinear Anal., 7(10):1127-1140, 1983.

[18] T. Cazenave. Semilinear Schrödinger equations, volume 10 of Courant Lecture Notes in Mathematics. New York University Courant Institute of Mathematical Sciences, New York, 2003.

[19] T. Cazenave and A. Haraux. Équations d'évolution avec non linéarité logarithmique. Ann. Fac. Sci. Toulouse Math. (5), 2(1):21-51, 1980.

[20] T. Cazenave and P.-L. Lions. Orbital stability of standing waves for some nonlinear Schrödinger equations. Comm. Math. Phys., 85(4):549-561, 1982.

[21] G. Ferriere. The focusing logarithmic Schrödinger equation: analysis of breathers and nonlinear superposition. Discrete Contin. Dyn. Syst., 40(11):6247-6274, 2020.

[22] G. Ferriere. Convergence rate in Wasserstein distance and semiclassical limit for the defocusing logarithmic Schrödinger equation. Anal. PDE, 14(2):617-666, 2021.

[23] P. Guerrero, J. López, and J. Nieto. Global solvability of the 3d logarithmic schrödinger equation. Nonlinear Analysis: Real World Applications, 11(1):79-87, 2010.

[24] G. A. Hagedorn. Semiclassical quantum mechanics. I. The $\hbar \rightarrow 0$ limit for coherent states. Comm. Math. Phys., 71(1):77-93, 1980

[25] G. A. Hagedorn. Semiclassical quantum mechanics. III. The large order asymptotics and more general states. Ann. Physics, 135(1):58-70, 1981.

[26] T. Hansson, D. Anderson, and M. Lisak. Propagation of partially coherent solitons in saturable logarithmic media: A comparative analysis. Phys. Rev. A, 80(3):033819, 2009.

[27] M. Hayashi. A note on the nonlinear Schrödinger equation in a general domain. Nonlinear Anal., 173:99$122,2018$.

[28] E. F. Hefter. Application of the nonlinear Schrödinger equation with a logarithmic inhomogeneous term to nuclear physics. Phys. Rev. A, 32:1201-1204, 1985.

[29] E. J. Heller. Time dependent approach to semiclassical dynamics. J. Chem. Phys., 62(1):1544-1555, 1975.

[30] K. Hepp. The classical limit for quantum mechanical correlation functions. Comm. Math. Phys., 35:265277, 1974.

[31] W. Krolikowski, D. Edmundson, and O. Bang. Unified model for partially coherent solitons in logarithmically nonlinear media. Phys. Rev. E, 61:3122-3126, 2000.

[32] S. D. Martino, M. Falanga, C. Godano, and G. Lauro. Logarithmic Schrödinger-like equation as a model for magma transport. Europhys. Lett., 63:472-475, 2003.

[33] T. C. Scott and J. Shertzer. Solution of the logarithmic Schrödinger equation with a Coulomb potential. $J$. Phys. Commun., 2(7):075014, 2018.

[34] J. Shertzer and T. C. Scott. Solution of the 3D logarithmic Schrödinger equation with a central potential. J. Phys. Commun., 4(6):065004, 2020.

[35] C. Villani. Topics in optimal transportation, volume 58 of Graduate Studies in Mathematics. American Mathematical Society, Providence, RI, 2003.

[36] F. B. Weissler. Logarithmic Sobolev inequalities for the heat-diffusion semigroup. Trans. Amer. Math. Soc., 237:255-269, 1978

[37] K. Yasue. Quantum mechanics of nonconservative systems. Annals Phys., 114(1-2):479-496, 1978.

[38] K. G. Zloshchastiev. Logarithmic nonlinearity in theories of quantum gravity: Origin of time and observational consequences. Grav. Cosmol., 16:288-297, 2010.

[39] K. G. Zloshchastiev. Spontaneous symmetry breaking and mass generation as built-in phenomena in logarithmic nonlinear quantum theory. Acta Phys. Polon. B, 42(2):261-292, 2011. 
Univ RENNES, CNRS, IRMAR - UMR 6625, F-35000 RENNES, FRANCE

Email address: Remi.Carles@math.cnrs.fr

Institut de Recherche Mathématique Avancée, UMR 7501 Université De Strasbourg et CNRS, 7 Rue RenÉ Descartes, 67000 Strasbourg, France

Email address: guillaume. ferriere@math.unistra.fr 